\title{
Human MFAP1 is a cryptic ortholog of the Saccharomyces cerevisiae Spp381 splicing factor
}

\author{
Alexander K. C. Ulrich ${ }^{1 *}$ and Markus C. Wahl $1^{1,2^{*}}$
}

\begin{abstract}
Background: Pre-mRNA splicing involves the stepwise assembly of a pre-catalytic spliceosome, followed by its catalytic activation, splicing catalysis and disassembly. Formation of the pre-catalytic spliceosomal B complex involves the incorporation of the U4/U6.U5 tri-snRNP and of a group of non-snRNP B-specific proteins. While in Saccharomyces cerevisiae the Prp38 and Snu23 proteins are recruited as components of the tri-snRNP, metazoan orthologs of Prp38 and Snu23 associate independently of the tri-snRNP as members of the B-specific proteins. The human spliceosome contains about 80 proteins that lack obvious orthologs in yeast, including most of the B-specific proteins apart from Prp38 and Snu23. Conversely, the tri-snRNP protein Spp381 is one of only five S. cerevisiae splicing factors without a known human ortholog.

Results: Using InParanoid, a state-of-the-art method for ortholog inference between pairs of species, and systematic BLAST searches we identified the human B-specific protein MFAP1 as a putative ortholog of the S. cerevisiae tri-snRNP protein Spp381. Bioinformatics revealed that MFAP1 and Spp381 share characteristic structural features, including intrinsic disorder, an elongated shape, solvent exposure of most residues and a trend to adopt a-helical structures. In vitro binding studies showed that human MFAP1 and yeast Spp381 bind their respective Prp38 proteins via equivalent interfaces and that they cross-interact with the Prp38 proteins of the respective other species. Furthermore, MFAP1 and Spp381 both form higher-order complexes that additionally include Snu23, suggesting that they are parts of equivalent spliceosomal sub-complexes. Finally, similar to yeast Spp381, human MFAP1 partially rescued a growth defect of the temperature-sensitive mutant yeast strain prp38-1.

Conclusions: Human B-specific protein MFAP1 structurally and functionally resembles the yeast tri-snRNP-specific protein Spp381 and thus qualifies as its so far missing ortholog. Our study indicates that the yeast Snu23-Prp38-Spp381 triple complex was evolutionarily reprogrammed from a tri-snRNP-specific module in yeast to the B-specific Snu23-Prp38-MFAP1 module in metazoa, affording higher flexibility in spliceosome assembly and thus, presumably, in splicing regulation.
\end{abstract}

Keywords: Alternative splicing, B-specific proteins, Pre-mRNA splicing, Spliceosome, U4/U6.U5, Tri-snRNP-specific proteins

\footnotetext{
* Correspondence: alexander.ulrich@fu-berlin.de; mwahl@zedat.fu-berlin.de

'Laboratory of Structural Biochemistry, Freie Universität Berlin, Takustr. 6,

D-14195 Berlin, Germany

Full list of author information is available at the end of the article
} 


\section{Background}

Splicing of primary transcripts is an essential step in the expression of many eukaryotic protein-coding genes. During splicing, non-coding intervening sequences (introns) are excised from a precursor (pre-) mRNA and neighboring coding regions (exons) are ligated via two consecutive transesterification reactions $[1,2]$. Pre-mRNA splicing is catalyzed by the spliceosome, a highly dynamic, multi-megadalton molecular ribonucleoprotein (RNP) machine that is composed of five small nuclear (sn) RNPs and numerous nonsnRNP proteins [3, 4]. For each round of splicing, a spliceosome is assembled in a stepwise fashion. The vast majority of splicing events in Saccharomyces cerevisiae $(s c)$ is constitutive and involves assembly of a spliceosome across an intron. In a constitutive splice event, U1 and U2 snRNPs recognize the 5'-splice site and branch point sequence of an intron, respectively, forming the A complex. Subsequently, the U4, U5 and U6 snRNPs join as a pre-formed tri-snRNP, giving rise to the pre-catalytic B complex. The B complex is then catalytically activated, yielding first the $\mathrm{B}^{\text {act }}$ and subsequently the $\mathrm{B}^{*}$ complex. The latter can carry out the first transesterification reaction of a splicing event. After step one of splicing, further rearrangements give rise to the $\mathrm{C}$ complex, which catalyzes the second transesterification reaction, subsequent to which the spliceosome is disassembled and subunits are recycled $[3,4]$.

Most primary transcripts in complex, multicellular eukaryotes contain more than one intron and can undergo alternative splicing to yield multiple mature mRNAs originating from the same gene [5]. The lengths of their introns vary considerably and can amount to several hundreds of thousands of nucleotides [6], while their exons are on average much shorter (ca. 120 nucleotides) and more homogeneous in size [7, 8]. Therefore, faithful localization of authentic 5'- and 3'-splice sites in complex, multicellular organisms is thought to occur via the initial assembly of spliceosomal complexes across exons (exon definition), which commits the pre-mRNA to the splicing pathway [9-12]. To allow intron excision, the interactions established during exon definition have to be reorganized to allow a 3'-splice site to be paired with an upstream 5'-splice site. Exon definition may proceed either to a cross-intron A complex [12] or directly to a cross-intron B complex under omission of a cross-intron A stage [13]. Functional pairing of specific splice sites, and thus the decision on a certain splicing pattern, is thought to take place during this conversion of cross-exon to cross-intron spliceosomal complexes [10, 14-16].

In yeast, pre-mRNA processing factor 38 domain containing protein (Prp38) and $23 \mathrm{kDa}$ small nuclear ribonucleoprotein component (Snu23) are integral components of the U4/U6.U5 tri-snRNP $[17,18]$, stay associated during tri-snRNP integration and B complex formation and leave the spliceosome again during the transition to the $\mathrm{B}^{\text {act }}$ complex [19]. The human orthologs of Prp38 and Snu23 are also exclusively present at the B complex stage but, in contrast to their yeast orthologs, associate with the pre-catalytic spliceosome independent of the tri-snRNP [20]. This feature they share with seven other non-snRNP proteins, collectively referred to as Bspecific proteins. The specific recruitment of B-specific proteins to the spliceosome during cross-exon to crossintron switching makes them prime candidates as regulators of alternative splicing.

Indeed, for most B-specific proteins there is evidence that they play a role in alternative splicing. In human, the group of B-specific proteins includes Prp38, Snu23, microfibrillar-associated protein 1 (MFAP1), suppressor of mec-8 and unc-52 protein homolog 1 (Smu1), Arg-Glu/Asp-repeat-containing protein (RED), forminbinding protein 21 (FBP21), $38 \mathrm{kDa}$ nuclear protein containing a WW domain (NPW38), NPW38-binding protein (NPW38BP) and ubiquitin-like protein 5 (UBL5) [20]. Homo sapiens (hs) Prp38 has acquired a veritable, Cterminal arginine-serine-rich (RS) domain, a hallmark of the splicing regulatory serine-arginine-rich (SR) proteins that are largely lacking in yeast [21]. UBL5, also called Hub1, was the first splicing factor that was found to be involved in alterative splicing in human [22] as well as in a rare case of alternative splicing in yeast [23]. In contrast, MFAP1, Smu1, RED, FBP21, NPW38 and NPW38BP lack obvious orthologs in yeast, where alternative splicing is essentially absent. MFAP1, Smu1 and RED have been implicated directly in the modulation of splice site choices in certain pre-mRNAs [24-29].

Presently, the precise functions of B-specific proteins are unknown. In particular, it is not clear to which extent they are important for both constitutive and alternative splicing, whether orthologs of some of these proteins are truly missing in yeast or have evolved so that they are not easily recognized or if yeast harbors other splicing factors that take over the constitutive roles of some of the B-specific proteins.

MFAP1 was first identified as a component of the extracellular matrix [30]. Later, the protein was found in spliceosome preparations [31], was shown to interact with Prp38 in pull-down experiments and to be required for pre-mRNA processing [32]. Interactions between MFAP1 and other B-specific proteins were identified by yeast twohybrid $(\mathrm{Y} 2 \mathrm{H})[21,33]$ and in vitro binding studies [21, 34]. Due to its elongated, solvent exposed nature and predicted dense array of short protein binding motifs, MFAP1 was suggested to act as a scaffold or ruler that engages multiple binding partners [34]. Recently, the molecular details of the MFAP1-Prp38 interaction have been revealed by Xray crystallography [34], representing one of the few 
structurally characterized interactions between B-specific proteins besides Snu23-Prp38 and Smu1-RED [35].

Here, we investigated whether $S$. cerevisiae contains an ortholog of metazoan MFAP1. Using InParanoid 8 and systematic BLAST searches, multiple sequence alignments, structure-guided interaction studies and a yeast growth assay, we identified the tri-snRNP-specific premRNA-splicing factor, suppressor of prp38-1 (Spp381), as the so far missing MFAP1 ortholog in S. cerevisiae.

\section{Results}

\section{Identification of MFAP1 orthologs in the eukaryotic tree of life}

To investigate when in eukaryotic evolution an MFAP1coding gene has been acquired, we conducted an ortholog search using the InParanoid 8 orthology analysis tool [36, 37]. The InParanoid methodology [38] uses pairwise BLAST-based all-versus-all sequence comparisons to detect orthologs in sets of protein-coding genes from 273 species (covering the major branches of the eukaryotic tree of life and selected prokaryotes), with each gene represented by one protein. To exclude false positive hits that merely arise from co-occurrence of abundant, highly conserved domains, InParanoid uses a strict cutoff criterion of sequence coverage $\geq 50 \%$ and BLAST score $\geq 50$. Taking into account the presumably low sequence conservation of MFAP1 due to the predicted structural disorder and the absence of folded protein domains [34], we also performed reciprocal best BLAST hit (RBH) searches of MFAP1 proteins against the same 273 sets of protein-coding genes with relaxed cut-off criteria (BLAST score $\geq 30$, E-value $\leq 0.01$ ). The RBH method has a relatively high specificity compared to other ortholog detection methods and its specificity is only marginally affected by changes in cut-off values [39].

The combined results of both analyses are presented in Fig. 1 and Additional files 1 and 2. An ortholog hit was classified as a high-confidence hit if identified by both methods (black boxes in Additional file 1). Hits delivered by one method alone were classified as medium confidence hits (grey boxes in Additional file 1). The case that the two methods identified two non-identical proteins as orthologous to the query did not occur. Our results show that $h s$ MFAP1 proteins are widely distributed in Metazoa (95.5\% or 84/88 of analyzed species), Ichthyosporea (100\%, 1/1), Choanoflagellida $(100 \%, 2 / 2)$, Amoebozoa (100\%, 4/4), Plantae (90\%, 18/20), SAR (Stramenopiles, Alveolates, Rhizaria; 88.9\%, 24/27), Fungi (without Ascomycota; 58.1\%, 18/31) and Ascomycota (without Saccharomycotina; 95.2\%, 40/42), but only sporadically present in Excavata $(25 \%, 2 / 8)$ and virtually absent in Saccharomycotina (without Saccharomycetaceae; $18.2 \%, 2 / 11)$ and in Saccharomycetaceae (0\%, 0/12). As expected, MFAP1 was not present in prokaryotes $(0 \%, 0 / 27)$ (Fig. 1). MFAP1 seems to be specifically absent in Saccharomycotina, as it is present in species that branched off from the human lineage much earlier in evolution than fungi (Amoebozoa ca. 1.5 billion years, Plantae ca. 1.5 billion years, Excarvates ca. 1.7 billion years, SAR ca. 1.8 billion years, fungi ca. 1.1 billion years; estimates obtained from timetree.org $[40,41])$ but also in many closely related Ascomycota species. In Saccharomycotina, MFAP1 was only found in Yarrowia lipolytica ( $y l$, UniProt ID: Q6CA21) and Wickerhamomyces ciferrii (wc, UniProt ID:

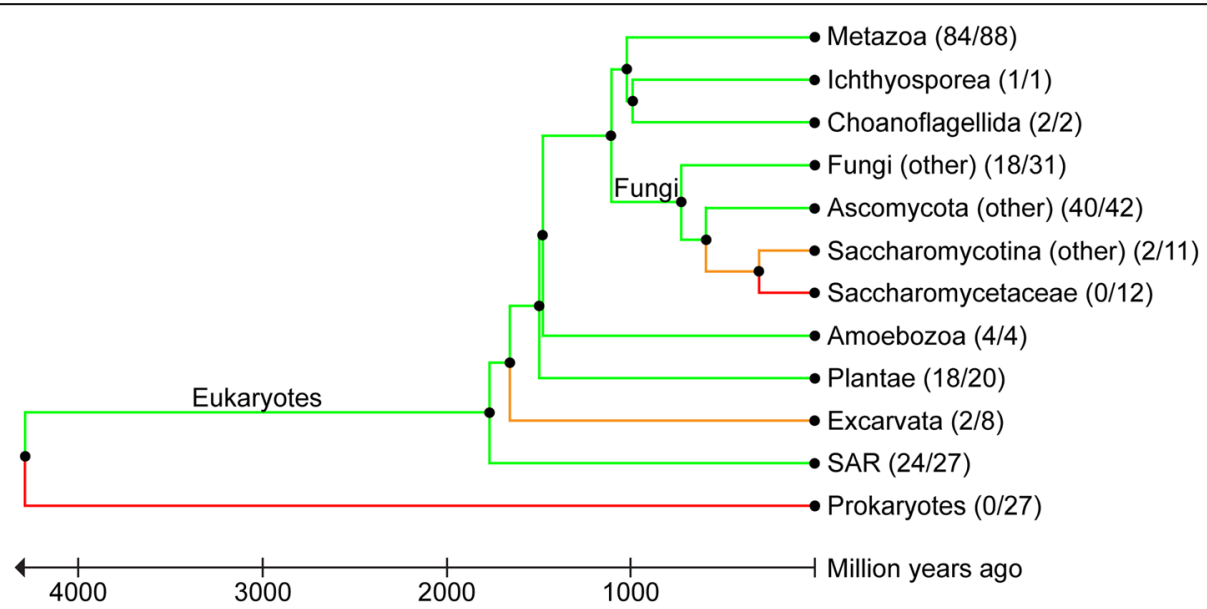

Fig. 1 Results summary of hsMFAP1 ortholog searches with InParanoid 8. The protein sequence of hsMFAP1 (UniProt ID: P55081) was used to search the InParanoid 8 [37] ortholog database and used as templates in BLAST searches against the 273 species (246 eukaryotes plus 27 prokaryotes) covered by the InParanoid 8 program. The phylogenetic tree is based on the divergence times of the taxonomic groups obtained from timetree.org $[40,41]$. The number of identified MFAP1 orthologs and the total number of analyzed species in respective taxonomic group is given in brackets. The branch color indicates the fraction of the analyzed species that contain an hsMFAP1 ortholog; green > 50\%, orange >0\% and $<50 \%$, red $0 \%$. See Additional file 1 for detailed results and Additional file 2 for UniProt IDs of identified orthologs 
K0KNQ2). Since the latter species is more closely related to Saccharomycetaceae, according to divergence time estimations by TimeTree.org ([40, 41]; wc: 212 MYA, $y l$ : 332 MYA), we performed an additional InParanoid ortholog search with wcMFAP1 as a query. We again identified orthologs in many metazoan $(78.4 \%, 69 / 88)$, fungal (excluding Ascomycota) $(48.4 \%, 15 / 31)$ and Ascomycota (excluding Saccharomycotina) species (81.0\%, 34/42). Hits identified in a species with both queries (hsMFAP1 and wcMFAP1) consistently resulted in the same protein. In addition, using $w c$ MFAP1 as a seed, we detected MFAP1 orthologs in all Saccharomycotina with the exception of Saccharomycetaceae (Additional files 1 and 2). These results indicate that MFAP1 orthologs are present in all major branches of the eukaryotic tree of life but appear to be absent in Saccharomyces cerevisiae and its close relatives (Saccharomycetaceae).

\section{Stepwise BLAST searches focused on the fungal kingdom identify Spp381 as a potential MFAP1 ortholog in Saccharomycetaceae}

To investigate whether Saccharomycetaceae have lost the mfap 1 gene or contain a highly diverged mfap 1 gene, we performed an MFAP1 ortholog search focused on the fungal kingdom with relaxed stringency. The results are summarized in Fig. 2, the raw data are presented in Additional file 3. For this purpose we performed BLAST searches with hsMFAP1 as a query against the proteomes of 103 fungal species that represent the fungal tree of life as published by Medina et al. [42]. This tree represents a consensus phylogeny combining three independent phylogenomic approaches (concatenated alignment, single- and multigene supertrees). Although there is a certain overlap between species selected by InParanoid and by Medina et al. and the total number of fungal species is similar (96 vs. 103), the phylogenetic tree by Medina et al. likely represents more accurate phylogenetic relationships among the fungi. To adapt the search to the low sequence similarity usually found between distant MFAP1 orthologs, we used the BLOSUM45 scoring matrix and selected for hits with BLAST score $\geq 30$, E-value $\leq 0.01$ and query coverage $\geq$ $20 \%$ (high confidence) or $\geq 10 \%$ (medium confidence). In addition, we required all further considered hits to represent the best hits in reverse BLAST searches.

As expected, MFAP1 orthologs were detected in the majority of non-Saccharomycotina fungi $(81.0 \%, 64 / 79)$, as well as in several non-Saccharomycetaceae Saccharomycotina species $(54.5 \%, 6 / 11)$, but were specifically absent in Saccharomycetaceae (0\%, 0/14). We assumed that if MFAP1 orthologs exist in Saccharomycetaceae, they would be evolutionary closest to neighboring Saccharomycotina species. Thus, we repeated the BLAST search with hsMFAP1 orthologs identified in the Saccharomycotina species Yarrowia lipolytica ( $y l$, UniProt ID:
Q6CA21), Pichia pastoris (pp, UniProt ID: A0A1B2J9D1), Debaryomyces hansenii (dh, UniProt ID: Q6BII8) and Candida albicans (ca, UniProt ID: C4YG44) against the 25 Saccharomycotina species of the Medina et al. fungal tree of life. All four species identified MFAP1 orthologs in the majority of non-Saccharomycetaceae Saccharomycotina species ( $y l: 7 / 11 ; p p: 10 / 11 ; d h: 9 / 11 ; c a: 11 / 11)$. In addition, all four also identified an MFAP1 ortholog in the Saccharomycetaceae organism Kluyveromyces lactis (Spp381, UniProt ID: Q6CJ60). Furthermore, Saccharomycetaceae MFAP1 orthologs were identified in Candida glabrata (UniProt ID: Q6FU95) by dhMFAP1 and in Lachancea thermotolerans by ppMFAP1, besides six medium confidence hits (query coverage $10-20 \%$ ) by ppMFAP1. We next selected $K$. lactis and C. glabrata MFAP1 orthologs as queries. Both queries identified orthologs in the same nine of 14 Saccharomycetaceae species, including Saccharomyces cerevisiae (Spp381, UniProt ID: P38282). In addition, the C. glabrata protein identified an ortholog in P. pastoris and K. lactis Spp381 found orthologs in most non-Saccharomycetaceae Saccharomycotina species (9/11). Finally, we performed the same analysis with the identified S. cerevisiae protein Spp381 as query and found orthologs in the same Saccharomycetaceae species (9/14) as with P. pastoris and C. glabrata in addition to one hit in non-Saccharomycetaceae Saccharomycotina (in P. pastoris). While the overall low sequence conservation of MFAP1 orthologs is especially pronounced between Saccharomycetaceae and neighboring Saccharomycotina species, the sequences of the MFAP1 orthologs of K. lactis (Spp381) and P. pastoris are able to bridge this gap.

To test if Spp381 proteins found in Saccharomycetaceae indeed represent a group of MFAP1 orthologs and not a different protein that coexists in MFAP1-containing nonSaccharomycetceae species, we used Spp381 from S. cerevisiae as a query in our InParanoid-based ortholog search (Fig. 1). ScSpp381 yielded orthologs in Mycospaerella graminicola, Sclerotinia sclerotiorum (both non-Saccharomycotina Ascomycota), Pichia pastoris (non-Saccharomycetaceae Saccharomycotina) - these proteins are the same as those identified in the initial search with hsMFAP1 - and in all twelve Saccharomycetaceae species. Thus, we did not find any non-MFAP1 protein as an $s c \mathrm{Spp} 381$ ortholog. These results show that Spp381 is not closely related, according to the InParanoid cut-off criteria, to any non-MFAP1 protein outside Saccharomycetaceae, indicating that Spp381 and MFAP1 do not coexist as different proteins in non-Saccharomycetaceae species. However, it is still possible that MFAP1 and Spp381 are highly similar proteins that emerged by convergent evolution and that exist in exactly complementary groups of organisms. It also cannot be excluded that MFAP1 and Spp381 might 


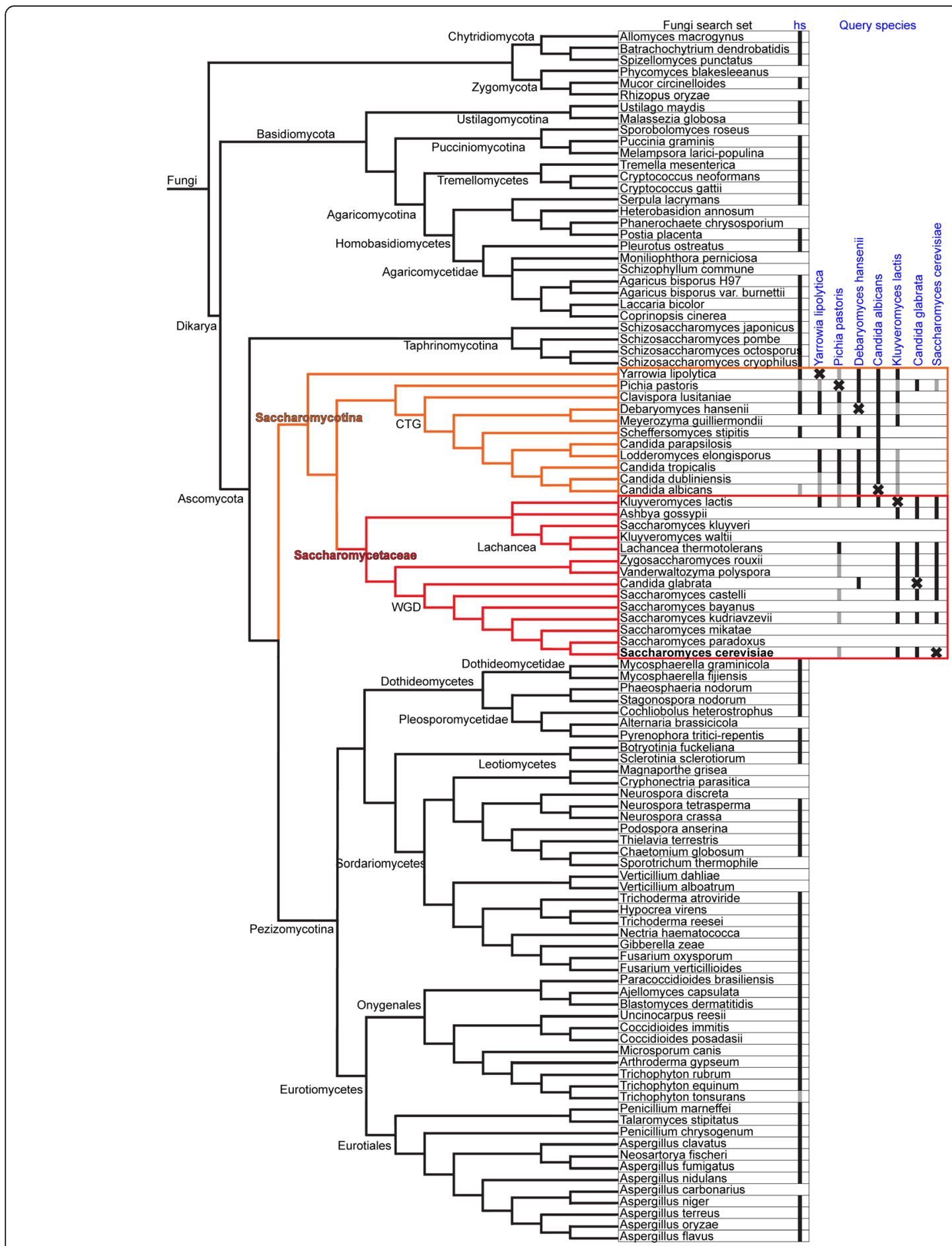

Fig. 2 Results of MFAP1 ortholog searches focused on the fungal kingdom. The protein sequence of Homo sapiens MFAP1 (UniProt ID: P55081) (hs) was used as template in BLAST searches against 103 fungi that represent the fungal tree of life as published by Medina et al. [42]. Seven MFAP1 orthologs identified in the Saccharomycotina subphylum, i.e. MFAP1 orthologs of Y. lipolytica (UniProt ID: Q6CA21), P. pastoris (UniProt ID: A0A1B2J9D1), D. hansenii (UniProt ID: Q6Bll8), C. albicans (UniProt ID: C4YG44), K. lactis (UniProt ID: Q6CJ60), C. glabrata (UniProt ID: Q6FU95) and S. cerevisiae Spp381 (UniProt ID: P38282), were then used as query sequences in further individual BLAST searches against the 25 Saccharomycotina species, including 14 Saccharomycetaceae species, that are part of the 103 fungal species. The identification of an ortholog within a species is indicated by black boxes (high confidence) or grey boxes (medium confidence). The fraction of the tree comprising Non-Saccharomycetaceae Saccharomycotina nodes is colored in orange; the tree fraction comprising Saccharomycetaceae nodes is colored in red. See Additional file 2 for raw data of BLAST searches 
have emerged from the same ancestral gene by duplication (paralogs) and that a different copy was lost in Saccharomycetaceae (mfap1) versus non-Saccharomycetaceae (spp381).

\section{S. cerevisiae Spp381 shares physicochemical, biochemical and structural features with $h$ sMFAP1}

The rather weak sequence similarity of Saccharomycetaceae Spp381 proteins to MFAP1 proteins (e.g. hsMFAP1 vs. scSpp381: 13.8\% identity, 27.4\% similarity; hsMFAP1 vs. klSpp381: $14.4 \%$ identity, $23.5 \%$ similarity) renders an orthology assumption difficult if based on primary sequence data alone. To further test the assumption that MFAP1 and Spp381 proteins are orthologs and not just randomly bestmatching proteins, we compared structural and functional data. Intriguingly, S. cerevisiae Spp381, like MFAP1, is a known splicing factor [43]. Moreover, scSpp381 had been identified by its ability to suppress defects elicited by the prp38-1 allele [43], which is associated with impaired spliceosome catalytic activation [44, 45], and its C-terminal half has been shown to directly interact with $s c \operatorname{Prp} 38$ in $\mathrm{Y} 2 \mathrm{H}$ assays [43], the ortholog of $h s \operatorname{Prp} 38$ that interacts with $h s$ MFAP1. Recent crystal structures of the hsPrp38hsMFAP1 complex (PDB ID: ID: 5F5S, Fig. 3a) and of the structurally highly similar Prp38-MFAP1 complex from the thermophilic fungus Chaetomium thermophilum ( $c t$; PDB ID: 5F5T, Fig. 3b) together with binding studies of arginineto-alanine mutants of the first and second arginine (R282, R286), which were sufficient to disrupt Prp38-MFAP1,

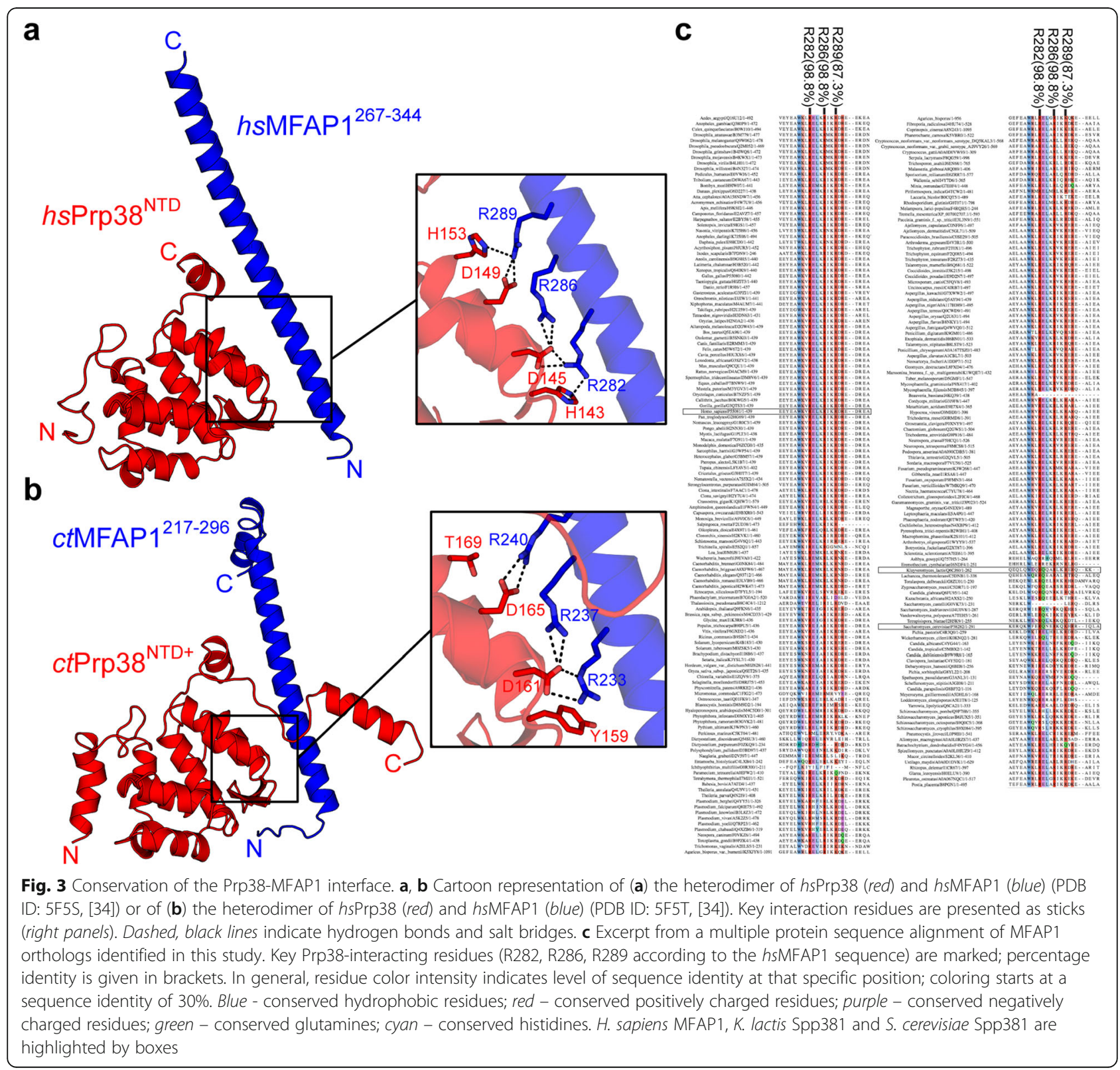


revealed a RxxxRxxR motif as a key Prp38-binding element of MFAP1 [34]. Strikingly, the C-terminal halves of $K$. lactis and S. cerevisiae Spp381 contain an identical or slightly modified motif, RxxxRxR and RxxxRxxK, respectively (Fig. 3c and Additional file 4). Among MFAP1 orthologs identified in this study, the first and second arginine residues are conserved in $98.8 \%$ and the third arginine in $87.3 \%$ of the cases, suggesting that most identified MFAP1 orthologs interact with Prp38 as well.

ScSpp381, hsMFAP1 and the two previously known MFAP1 orthologs ctMFAP1 [34] and C. elegans (ce) MFAP1 [25] also share a number of physicochemical and biochemical properties, i.e. (1) a high fraction of charged residues (scSpp381 39.2\%; hsMFAP1 46.5\%, ctMFAP1 49.9\%, ceMFAP1 50.9\%; UniProt average 23.6\%); (2) a low isoelectric point (scSpp381 5.4; hsMFAP1 5.0, ctMFAP1 6.7, ceMFAP1 4.9); (3) an increased apparent molecular mass on SDS-PAGE (35 kDa $s c S p p 381$ running at ca. $50 \mathrm{kDa}$; $52 \mathrm{kDa} h s \mathrm{MFAP} 1,52 \mathrm{kDa} c t \mathrm{MFAP} 1$ and $56 \mathrm{kDa} c e \mathrm{MFAP} 1$ running at ca. $75 \mathrm{kDa}$ ); and (4) PEST elements, which are expected to reduce the half-lives of the proteins [46], with PEST-scores $>16$ (scSpp381 residues 56-95, PEST-score +29.8; hsMFAP1 residues $67-85$ and 174-198, PESTscores +27.5 and +25.1; ctMFAP1 residues 21-44, 105-140 and 161-178, PEST-scores $+19.1,+35.6$ and +18.7; ceMFAP1 residues 192-223, PEST-score +35.2 [47]). In a multiple sequence alignment of all identified MFAP1 orthologs, the PEST element of $s c$ Spp381 aligns with PEST elements hsMFAP1 174-198, ctMFAP1 105-140 and ceMFAP1 192-223. In addition, the proteins are predicted to share a similar structure in isolation, i.e. they are predicted to be intrinsically disordered (scSpp381 99.7\%, hsMFAP1 97.3\%, ctMFAP1 100.0\%, ceMFAP1 98.8\%; average human protein 21.6\%; average S. cerevisiae protein $17.0 \%$ [48]), with most residues solvent exposed ( $s c$ Spp381 90.4\%, hsMFAP1 86.6\%, ctMFAP1 66.7\%, ceMFAP1 84.1\%) and with a tendency to form $\alpha$-helices (scSpp381 32.0\%, hsMFAP1 72.9\%, ctMFAP1 47.0\%, ceMFAP1 62.9\%; average globular protein $30 \%$ [49]) (Fig. 4a). Structural disorder of $h s$ MFAP1
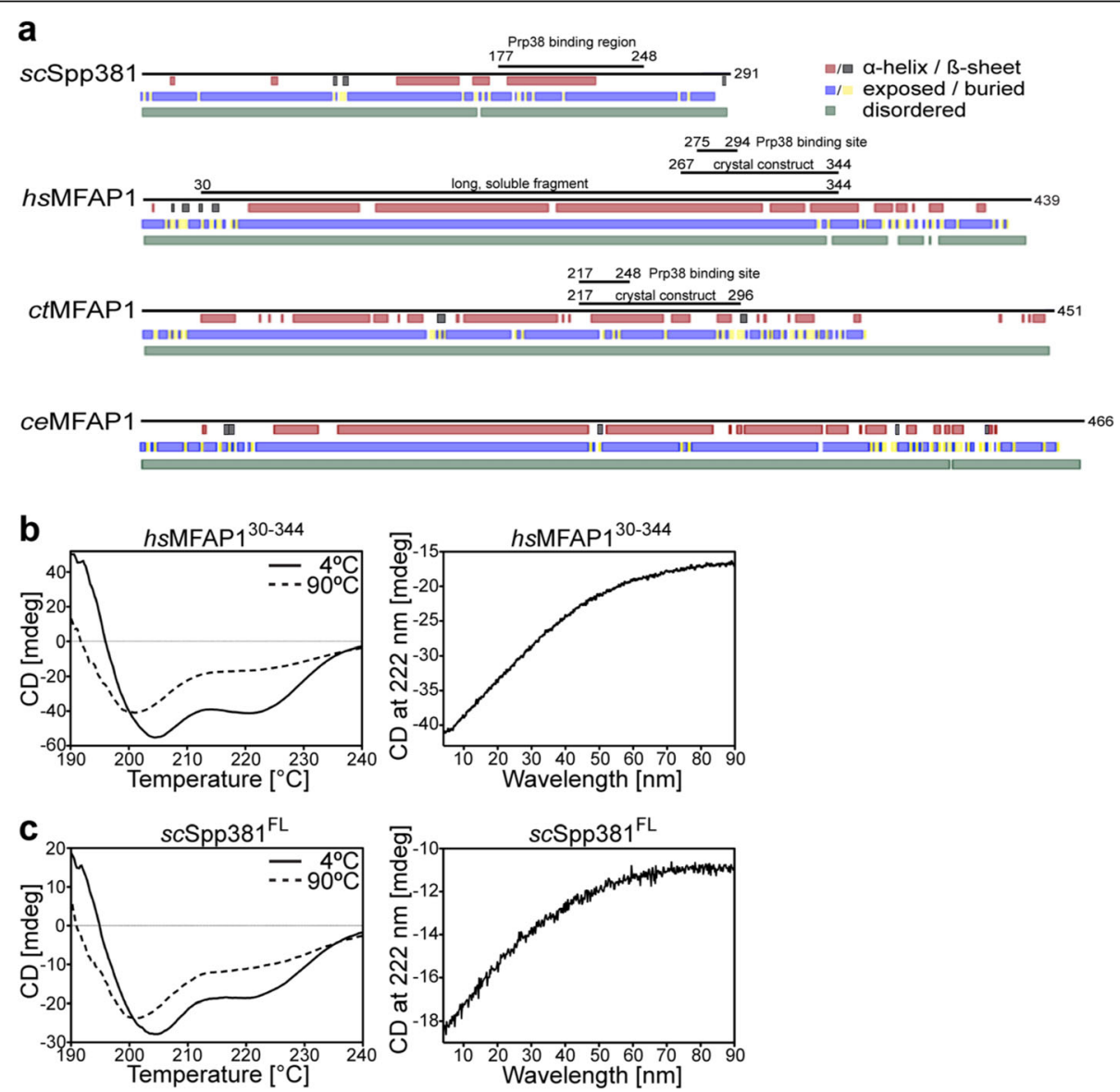

Fig. 4 Domain organization and CD spectra of MFAP1 and Spp381. a Secondary structure prediction and domain organization of Spp381 from S. cerevisiae and of MFAP1 from H. sapiens, C. thermophilum and C. elegans. Red/gray bars - predicted a-helices/ß-strands; blue/yellow bars - predicted solvent exposed/buried regions; green bars - regions of predicted structural disorder. b, c CD spectra of hsMFAP1 and scSpp381. CD spectra $(\lambda=190-$ $240 \mathrm{~nm}$ ) at $4{ }^{\circ} \mathrm{C}$ (solid lines) and $90^{\circ} \mathrm{C}$ (dashed lines) and CD melting curves $\left(4-90^{\circ} \mathrm{C}\right.$ ) at $222 \mathrm{~nm}$ of (b) hsMFAP1 ${ }^{30-344}$ and of (c) full-length scSpp381 
and $s c \operatorname{Spp} 381$ was also previously predicted in independent studies [50, 51]. Indeed, CD spectroscopy showed that $s c \operatorname{Spp} 381$, like $h s$ MFAP1, has significant $\alpha-$ helical content in isolation, which gradually changes to a more random coil structure upon heating with no sharp transition (Fig. 4b and c), indicative of a lack of a stable tertiary fold. Irrespective of the exact evolutionary relationship of yeast Spp381 and metazoan MFAP1 proteins, the above analyses indicate that both proteins are structurally very similar and share a Prp38-binding motif.

\section{Interaction studies corroborate similar functions of scSpp381 and hsMFAP1}

ScSpp381 shares the ability of $h s$ MFAP1 to bind Prp38, as shown by $\mathrm{Y} 2 \mathrm{H}$ analyses [21, 43]. We confirmed this interaction with isolated, recombinant, wild type $s c \operatorname{Prp} 38$ and $s c \mathrm{Spp} 381$ proteins that co-migrated on a gel filtration column (Fig. 5a). To further test if this interaction also uses the same interface as reported in the human and $C$. thermophilum Prp38-MFAP1 complexes [34], we introduced point-mutations into $s c \operatorname{Prp} 38$ and $s c$ Spp381 corresponding to complex-disrupting point-mutations in human Prp38 and MFAP1 (Fig. 3a) and tested interaction of the proteins by analytical gel filtration. Analogous to the Prp38-MFAP1 complexes [34], a D189A mutation in $s c \operatorname{Prp} 38$ (corresponding to D145A in $h s \operatorname{Prp} 38$ ) as well as a R192A mutation in $s c S p p 381$ (corresponding to R282A in hsMFAP1) led to disruption of the complex (Fig. 5b). Furthermore, $s c S p p 381^{177-248}$, corresponding to $h s \mathrm{MFAP} 1^{267-}$ 344 , the minimal MFAP1 fragment used for crystallization of the $h s$ Prp38-hsMFAP1 complex [34], was sufficient to bind $s c \operatorname{Prp} 38$ (Fig. 6a), further underlining the structural and functional similarities. In addition, we could assemble a trimeric $s c$ Snu23 $3^{116-169}-s c \operatorname{Prp} 38-s c S p p 381^{177-248}$ complex (Fig. 6b), resembling the minimal trimeric Snu23-Prp38MFAP1 complex in the thermophilic fungus C. thermophilum, of which the crystal structure has been solved [34]. These results indicate that $s c \mathrm{Spp} 381$ and $h s \mathrm{MFAP} 1$ bind their respective Prp38 partners via equivalent interfaces and via the same key residues, and that Spp381 is involved in the same trimeric complex as MFAP1.

To investigate if the structural similarity between $s c \mathrm{Spp} 381$ and $h s \mathrm{MFAP} 1$ is high enough so that they can substitute for each other in Prp38 binding, we performed cross-species interaction studies. Indeed, $s c \operatorname{Prp} 38$ bound $h s$ MFAP1 ${ }^{267-344}$ (Fig. 7a) and $h s \operatorname{Prp} 38^{\mathrm{NTD}+}$, lacking the complex, multicellular organism-specific RS domain, stably interacted with $s c \mathrm{Spp} 381$ (Fig. 7b). The latter interaction did not form with the D145A variant of $h s \operatorname{Prp} 38^{\mathrm{NTD}+}$ (Fig. 7b). These results show that Spp381 and MFAP1 can substitute for each other in spliceosomal complexes and thus might share a similar interaction network in the spliceosome.
Human MFAP1 can partially substitute for yeast Spp381 in its function to rescue the conditionally lethal mutant yeast strain prp38-1

The conditionally lethal yeast strain prp38-1 produces a mutant version of the Prp38 protein and displays a growth defect at $37{ }^{\circ} \mathrm{C}$ [44]. Expression of plasmidencoded wild type $s c \operatorname{Prp} 38$ but also of $s c \mathrm{Spp} 381$ efficiently suppresses this growth defect [43]. To test if $h s$ MFAP1 can exploit its capability to bind $s c \operatorname{Prp} 38$ in a $s c$ Spp381-like manner to also functionally substitute for scSpp381 in vivo, we performed yeast growth assays. As expected, all tested prp38 and prp38-1 strains grew equally well at $23^{\circ} \mathrm{C}$ (Fig. 8, left panel). At $37^{\circ} \mathrm{C}$ (Fig. 8, right panel), wild type prp38 displayed slightly reduced growth compared to $23{ }^{\circ} \mathrm{C}$ (row 1). As previously reported, prp38-1 showed complete growth arrest at $37^{\circ} \mathrm{C}$ (row 2). Growth of prp38-1 at $37^{\circ} \mathrm{C}$ was largely restored by transformation with a plasmid encoding wild type scPrp38 (YEp13-2, row 3), partially restored by plasmids encoding scSpp381 (YEp13-7 and YEplac112-7A, rows 4-5) and weakly restored by a plasmid encoding hsMFAP1 (YEplac112-MFAP1, row 6). Although expression of plasmid-encoded $h s$ MFAP1 did not suppress prp38-1 as efficiently as over-production of $s c \mathrm{Spp} 381$, we conclude that $h s$ MFAP1 can fulfill certain Prp38supporting functions of $s c \operatorname{Spp} 381$ in yeast.

\section{Discussion \\ HsMFAP1 is a cryptic ortholog of the yeast splicing factor Spp381}

Proteomics analyses revealed that almost all factors required for constitutive splicing in S. cerevisiae are also present in human spliceosomes [4, 19]. Presently, yeast proteins with missing human orthologs include the U1 factors Prp42 and Snu56, the Prp19-associated complex protein Ntc20, the disassembly factor Ntr2 [19] and the U4/U6.U5 tri-snRNP-specific protein Spp381. Compared to yeast, human spliceosomes include $\sim 80$ additional, predominantly non-snRNP proteins, whose precise functions during splicing are in many cases unclear $[4,19]$.

Here, we applied the ortholog detection tool InParanoid 8 as well as stepwise BLAST searches to identify MFAP1 as a likely ortholog of the $S$. cerevisiae trisnRNP-specific protein Spp381. By phyletic profiling we unambiguously identified MFAP1 orthologs in nearly all major branches of the eukaryotic tree of life, including in organisms that split from the common lineage with multicellular eukaryotes about 1.8 billion years ago [40, 41], with the exception of Saccharomycetaceae, that separated 1.1 billion years ago [40, 41] (Table 1), where stepwise BLAST searches instead uncovered the Spp381 protein. The evolutionary relationship between MFAP1 and Spp381 was further supported by strong structural similarities between $h s$ MFAP1 and $s c$ Spp381 that would 
a
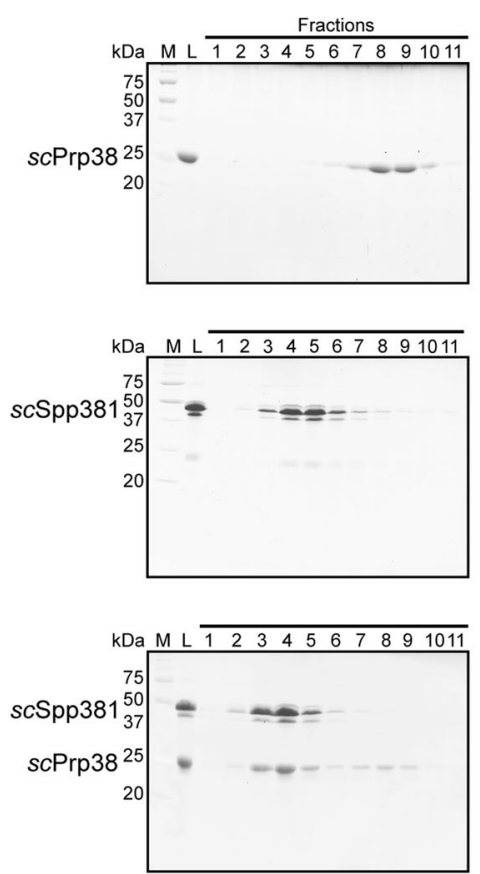

b
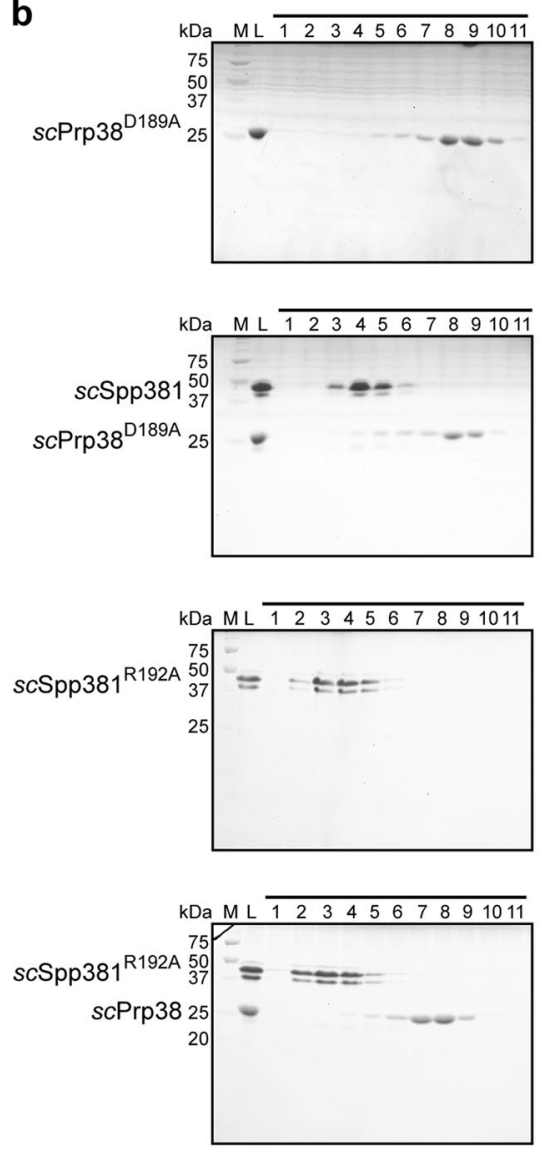
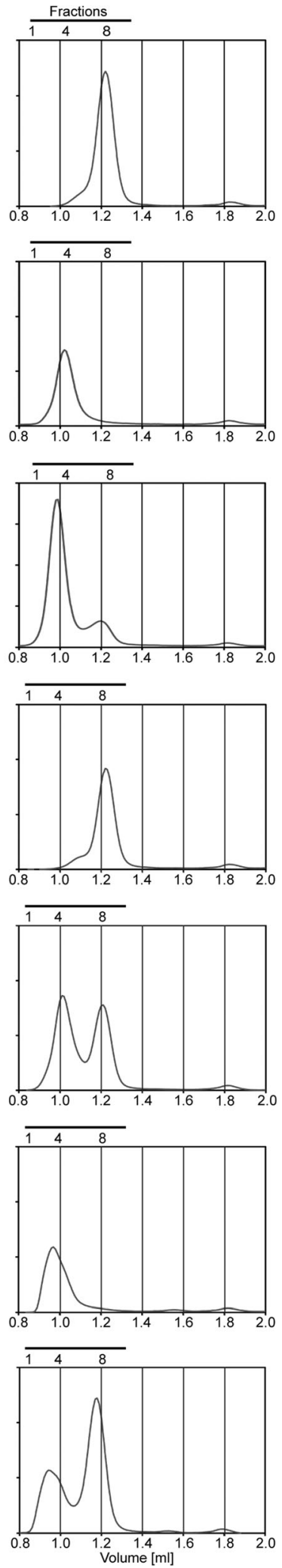

Fig. 5 (See legend on next page.) 
(See figure on previous page.)

Fig. 5 The Prp38-MFAP1/Spp381 interface is conserved between yeast and human. Coomassie Blue-stained SDS-PAGE gels and corresponding chromatograms of analytical gel filtration experiments with full-length scPrp38 and scSpp381. a Wild type proteins. b Wild type scSpp381 in combination with the scPrp38 D189A variant or wild type scPrp38 in combination with the scSpp381 R192A variant. All experiments were performed with a Superdex 75 3.2/30 column (GE Healthcare). M - marker; L - load; horizontal black lines - fractions analyzed by SDS-PAGE

allow Spp381 to fulfill a role as a flexible scaffolding factor as proposed for MFAP1 [34]. Finally, we presented two key functional indications supporting the assumed evolutionary connection. First, our interaction studies with wild type proteins, single point mutants that failed to interact and cross-species interactions between $h s \operatorname{Prp} 38 / s c \operatorname{Prp} 38$ and $h s \mathrm{MFAP} 1 / s c S p p 381$, showed that the $s c \operatorname{Prp} 38-s c S p p 381$ complex is established via a very similar interface to the one observed in the recently structurally characterized $h s$ Prp38-hsMFAP1 complex [34]. Although we cannot completely rule out the possibility that $h s$ MFAP1 and $s c S p p 381$ evolved independently to bind the same surface on Prp38, it is rather unlikely that in this case both interactions would rely on exactly corresponding residues. In addition, MFAP1 and Spp381 both bind Prp38 in the context of a trimeric complex with Snu23, further increasing the likelihood of an evolutionary relationship between MFAP1 and Spp381. Second, hsMFAP1, like scSpp381, weakly suppresses the temperature-induced growth defect of yeast strain prp38-1, most likely by interacting with and stabilizing the mutated Prp38 protein, suggesting that $h s$ MFAP1 can fulfill certain $s c \operatorname{Spp} 381$ functions in vivo. We acknowledge the possibility that a a
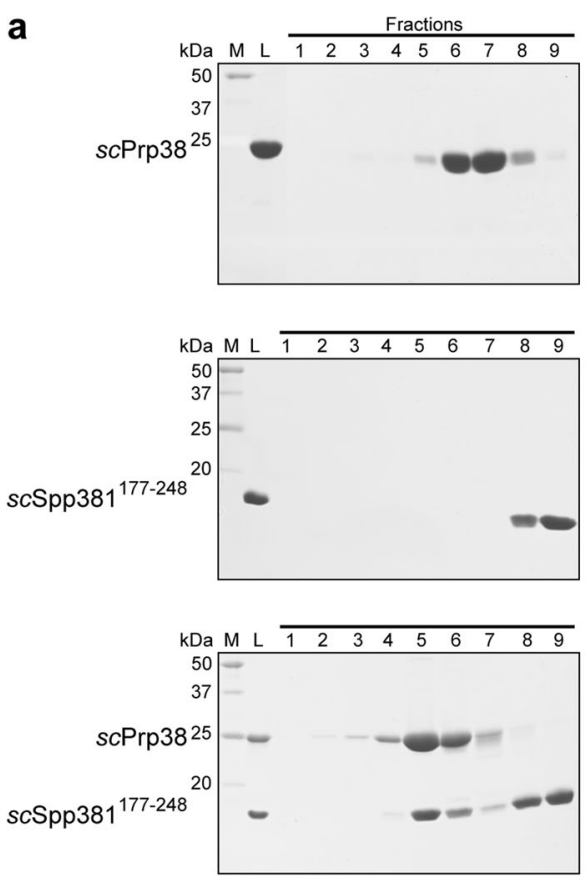

b

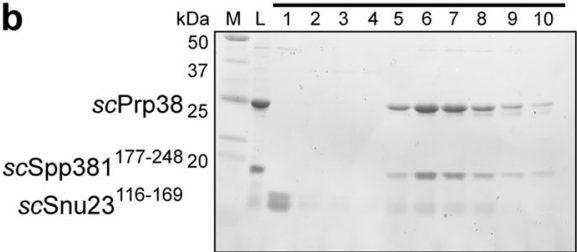

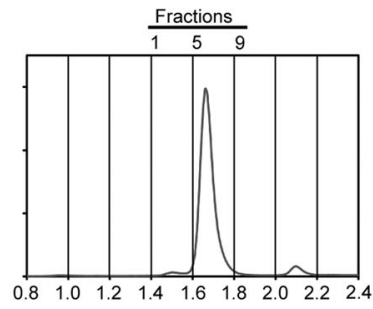
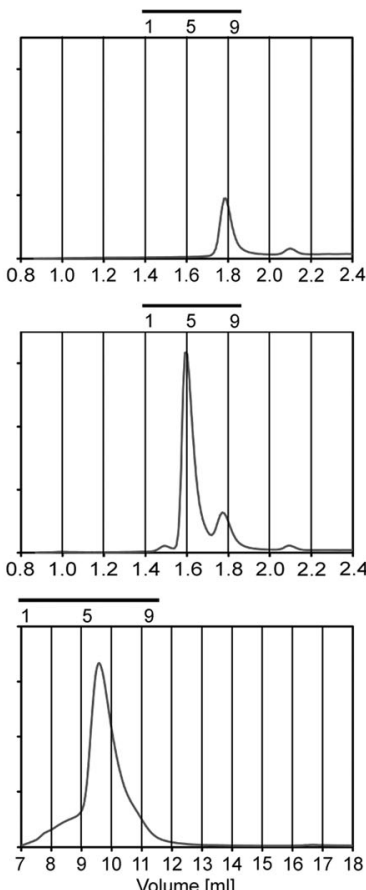

Fig. 6 Minimal scPrp38-scSpp381 and scSnu23-scPrp38-scSpp381 complexes resemble analogous MFAP1-based complexes in H. sapiens and C. thermophilum. Coomassie Blue-stained SDS-PAGE gels and corresponding chromatograms of analytical gel filtration experiments with the indicated scPrp38 and scSpp381 (a), or with the indicated scPrp38, scSpp381 and scSnu23 variants (b). Experiments in (a) were performed with a Superdex 200 increase 3.2/300 column. Experiments in (b) were carried out on a Superdex 75 10/300 column (both GE Healthcare). M - marker; L - load; horizontal black lines - fractions analyzed by SDS-PAGE 
a
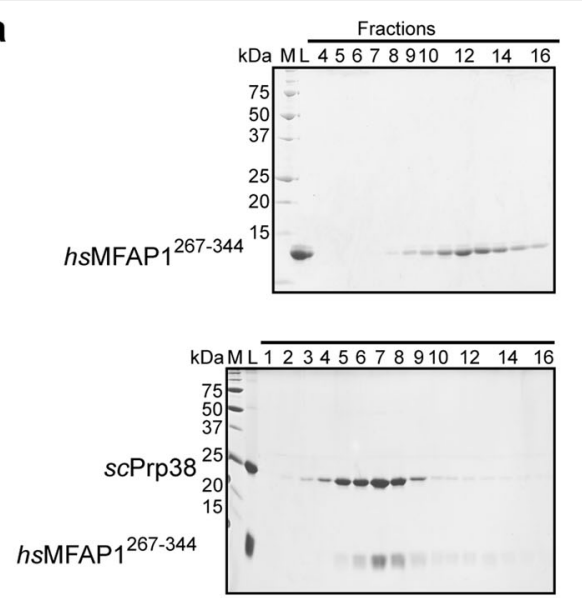

b
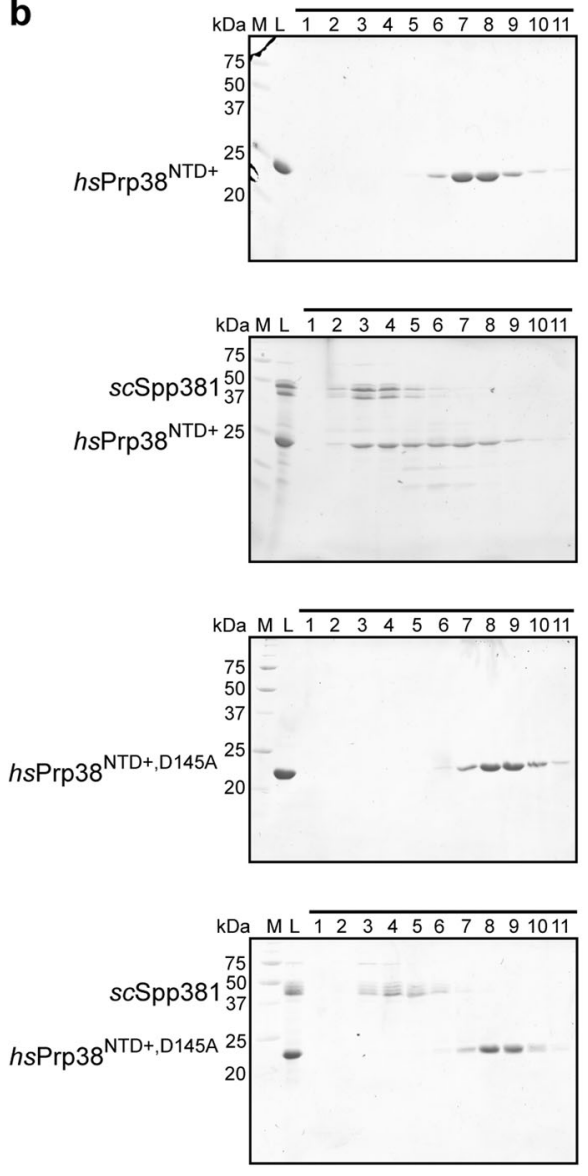
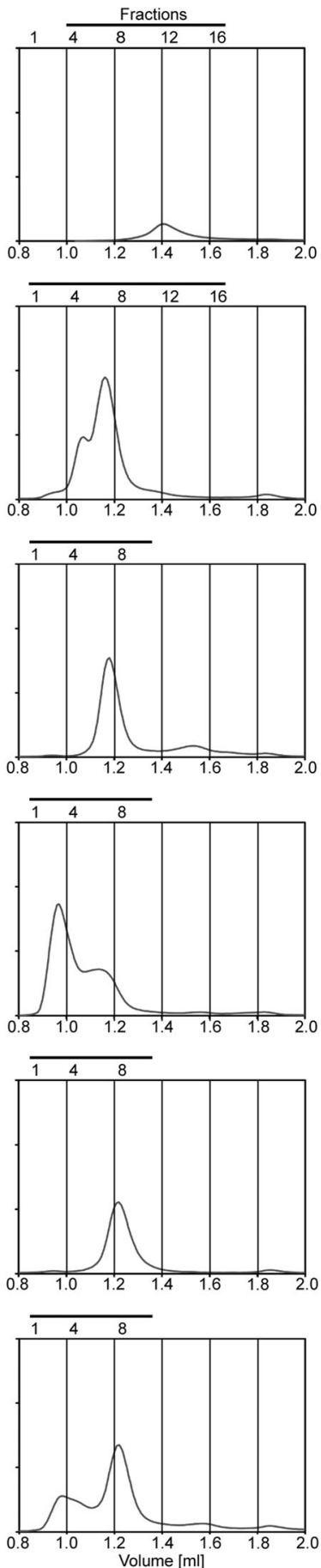

Fig. 7 Cross-species interaction studies. Coomassie Blue-stained SDS-PAGE gels and corresponding chromatograms of analytical gel filtration experiments. a ScPrp38 binds hsMFAP1 ${ }^{267-344}$, a minimal hsPrp38-interacting fragment. b HsPrp38 ${ }^{\mathrm{NTD}+}$, but not hsPrp38 ${ }^{\mathrm{NTD}+, \mathrm{D} 145 \mathrm{~A}}$, binds scSpp381. All experiments were performed with a Superdex 75 10/300 column (GE Healthcare). M - marker; L - load; horizontal black lines - fractions analyzed by SDS-PAGE

protein that is evolutionarily unrelated to Spp381 might also be able to bind and stabilize the mutated Prp38 protein in prp38-1. However, the ability to rescue this growth defect likely requires a set of specific features, including a specific binding mode to Prp38, certain physicochemical properties and the ability to interact with additional binding partners, that seem be overlapping between $h s$ MFAP1 and $s c \operatorname{Spp} 381$ to a large degree and are unlikely to be 


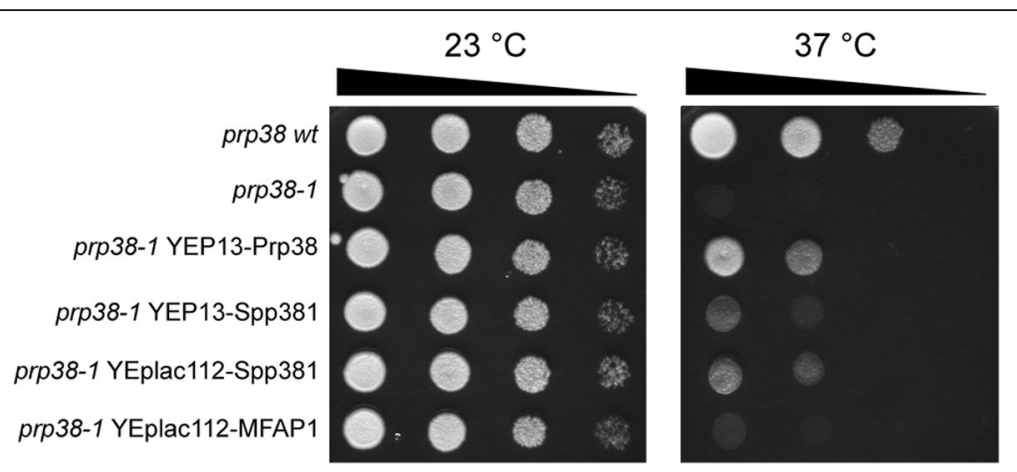

Fig. 8 scSpp381 and hsMFAP1 partially suppress the growth defect of temperature sensitive yeast strain prp38-1. YPD-agar plates were incubated for 3 days at 23 or $37^{\circ} \mathrm{C}$. Each row contains serial dilutions (initial $\mathrm{OD}_{600}: 2.0,0.2,0.02$, and 0.002 ) of the indicated yeast strains. Experiments were conducted in triplicates; representative examples are shown

shared by unrelated proteins. The reduced level of suppression by $h s$ MFAP1 compared to $s c S p p 381$ might be explained by a lower expression level of plasmid-encoded hsmfap1 compared to plasmid-encoded scspp381 in the prp38-1 strain context, a potentially tighter interaction of scPrp38-scSpp381 versus scPrp38-hsMFAP1 and/or the inability of $h s$ MFAP1 to bind one or more binding partners of $s c$ Spp381 other than Prp38 in yeast. The latter two possibilities are supported by the nature of the proteinbinding sites of MFAP1 and Spp381; they comprise short, peptide motif-like sequences with limited structural restraints [34]. Thus, the binding sites are highly likely, over the course of evolution, to strongly adapt to their diverging interaction partners. This notion is in agreement with the overall low sequence similarity between $s c \operatorname{Spp} 381$ and hsMFAP1.

Taken together, the sequence similarity between MFAP1 and SPP381 does not suffice to delineate their precise evolutionary relationships. Yet, they are structurally and functionally similar to an extent that they can substitute for each other. This suggests that, indeed, both proteins may represent orthologs although other evolutionary scenarios cannot be entirely ruled out.

\section{Functional characteristics of MFAP1 and Spp381 proteins may allow for high evolutionary rates of sequence divergence}

Identification of a common evolutionary origin of proteins by sequence comparisons is increasingly challenging with decreasing sequence conservation. Fast diverging sequences lack the evolutionary pressure commonly associated with the maintenance of a particular 3D fold or of extended interaction surfaces. The human B-specific protein MFAP1 is characterized by a lack of stable tertiary structure, structural flexibility and relatively short, but nevertheless high-affine, protein-protein interaction sites and plays a role as an elongated scaffolding factor that could transmit conformational changes within the

Table 1 Summary of ortholog analyses

\begin{tabular}{|c|c|c|c|c|c|c|c|c|c|}
\hline \multirow[t]{3}{*}{ Search set ${ }^{a}$} & \multicolumn{9}{|c|}{ Query species $^{\mathrm{b}}$} \\
\hline & \multirow[b]{2}{*}{ hs } & \multicolumn{5}{|c|}{ Saccharomycotina } & \multicolumn{3}{|c|}{ Saccharomycetaceae } \\
\hline & & $W C$ & $y l$ & $p p$ & $d h$ & $\mathrm{ca}$ & $k l$ & $\mathrm{cg}$ & SC \\
\hline Eukaryotes (other) $^{c}$ & $+/+/ 0$ & $+/+/ 0$ & $\mathrm{O} / \mathrm{O} / \mathrm{O}$ & $\mathrm{O} / \mathrm{O} / \mathrm{O}$ & o/০/০ & $\mathrm{O} / \mathrm{O} / \mathrm{O}$ & O/O/O & O/O/O & $-/-/ 0$ \\
\hline Fungi (other) $^{d}$ & $+/+/+$ & $+/+/ 0$ & O/O/O & $\mathrm{O} / \mathrm{O} / \mathrm{O}$ & $\mathrm{O} / \mathrm{O} / \mathrm{O}$ & $\mathrm{O} / \mathrm{O} / \mathrm{O}$ & O/০/O & O/O/O & $-/-/ 0$ \\
\hline Ascomycota (other) ${ }^{e}$ & $+/+/+$ & $+/+/ 0$ & ০/০/০ & O/০/O & O/O/O & $\mathrm{O} / \mathrm{O} / \mathrm{O}$ & ০/০/০ & O/O/O & $-1+/ 0$ \\
\hline Saccharomycotina ${ }^{f}$ & $+/+/+$ & $+/+/ 0$ & $\mathrm{O} / \mathrm{O} /+$ & O/O/+ & $\mathrm{O} / \mathrm{O} /+$ & $\mathrm{O} / \mathrm{O} /+$ & $\mathrm{O} / \mathrm{O} /+$ & O/O/+ & $-/+/+$ \\
\hline Saccharomycetaceae & $-/-/-$ & $-/-/ 0$ & $\mathrm{O} / \mathrm{O} /+$ & $\mathrm{O} / \mathrm{O} /+$ & $\mathrm{O} / \mathrm{O} /+$ & $\mathrm{O} / \mathrm{O} /+$ & $\mathrm{O} / \mathrm{O} /+$ & $\mathrm{O} / \mathrm{O} /+$ & $+/+/+$ \\
\hline
\end{tabular}

____ Search strategy: InParanoid database search/InParanoid BLAST search/fungi-focused BLAST search

+ At least one MFAP1 ortholog was identified in the respective search set

- No MFAP1 ortholog was identified in the respective search set

o Search set was not used in the respective analysis

${ }^{a}$ Taxomeric group that was used as the search set

${ }^{b}$ MFAP1 ortholog of this species was used as a search query

'Eukaryotes other than fungi

${ }^{\mathrm{d}}$ Fungi other than Ascomycota

eAscomycota other than Saccharomycotina

fSaccharomycotina other than Saccharomycetaceae 
spliceosome [34]. These functional characteristics likely allow for a high sequence divergence rate during evolution, in particular in regions of the protein that only require the maintenance of an elongated, flexible structure.

Indeed, the sequence identity between known MFAP1 orthologs is low and even less recognizable for evolutionary distant MFAP1 orthologs identified in our study (Additional file 5). In this context it is not surprising that MFAP1 and Saccharomycetaceae Spp381 sequences also exhibit a low sequence identity. More surprising, however, is the low sequence conservation between Saccharomycotina and other Ascomycota species, between Saccharomycotina and Saccharomycetaceae, and even between neighboring Saccharomycetaceae organisms (Additional file 5).

\section{Liberation from the tri-snRNP may enable B-specific proteins} to perform their functions in a regulated manner

In addition to the large number of human splicing factors that do not have an obvious conserved counterpart in yeast [4, 19], "reprogramming" of splicing factors Prp38 and Snu23 from stable snRNP components in yeast to non-snRNP proteins in human (Fig. 9) illustrates a lower level of fixed pre-organization of metazoan spliceosomes, even with respect to core splicing factors. In yeast, $s c \operatorname{Prp} 38$ and $s c$ Snu23 are recruited at the same time and with the same efficiency as all other U4/U6.U5 tri-snRNP components to cross-intron spliceosomal A complexes [19]. While their precise roles during spliceosome activation are still unclear, it is obvious that in a situation as encountered in yeast, there is no possibility to regulate, for example, the kinetics of spliceosome activation via a more or less efficient recruitment of Prp38 or Snu23 compared to other tri-snRNP components. The situation is decisively different in metazoa, where Prp38 and Snu23 are non-snRNP proteins (Fig. 9) [52]. While they are still recruited at the stage of B complex formation, irrespective of whether the $\mathrm{B}$ complex originated from a cross intron A complex [20] or a crossexon complex [13], their binding could, in principle, be regulated independent of the binding of the U4/U6.U5 tri-snRNP. Thus, while e.g. Prp38 most likely can influence the efficiency of catalytic activation also in complex, multicellular eukaryotes [32], the timing of when it unfolds this activity could differ, for example, in two competing alternative splicing situations (which may exhibit different compositions, conformations or spatial distributions of components). Differential binding of Prp38 and Snu23 could thus promote catalytic activation of two competing spliceosomal complexes with a different efficiency and thus influence the relative frequency with which mutually exclusive splice sites are used.

Our findings suggest that a similar functional relationship as between yeast and metazoan Prp38 and Snu23

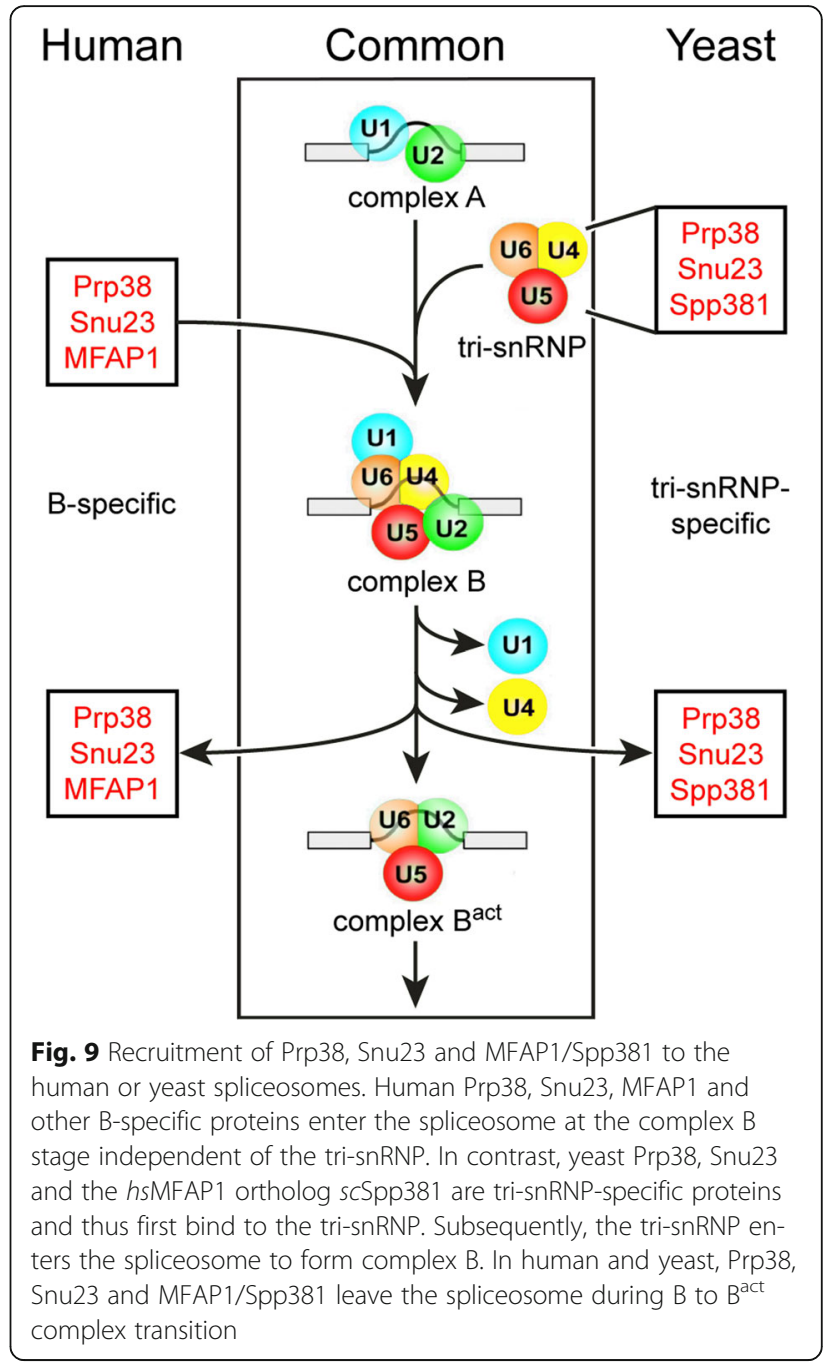

proteins [21] exists between yeast Spp381 and metazoan MFAP1 proteins. As disruption of the scspp381 gene leads to severe growth defects and accumulation of unspliced pre-mRNA in vivo [43], scSpp381 is an important, albeit not essential, splicing factor that apparently acts in the same process as $s c \operatorname{Prp} 38$. We showed that $s c \mathrm{Spp} 381$ and $h s$ MFAP1 exhibit cross-species interactions with the respective Prp38 proteins, suggesting that MFAP1 may be responsible for Spp381-like functions in complex, multicellular eukaryotes. During functional pairing of splice sites after initial cross-intron or cross-exon spliceosome assembly, spliceosomes face the problem of locating and bringing together spliceosomal subunits that are bound at the intron ends and thus may be spatially separated [3]. Elongated proteins that are specifically recruited at this stage, such as $h s$ MFAP1 and scSpp381, are well suited to help align and gather spatially separated parts of the spliceosome. They could serve as scaffolds or rulers, e.g. during functional pairing of splice sites, by using limited-length binding epitopes 
arrayed along their sequence to engage multiple binding partners [34]. However, like $s c \operatorname{Prp} 38$ and $s c S n u 23$, $s c \mathrm{Spp} 381$ is a stable component of the U4/U6.U5 trisnRNP $[17,18,43]$, while $h s$ MFAP1, like $h s \operatorname{Prp} 38$ and $h s \mathrm{Snu} 23$, is a non-snRNP B-specific protein [20] (Fig. 9). As in the case of yeast and metazoan Prp38 and Snu23, the tri-snRNP nature of $s c \operatorname{Spp} 381$ mandates that it is always recruited to spliceosomal B complexes together with other tri-snRNP components, thus rendering its function constitutive. In contrast, the non-snRNP, B-specific MFAP1 protein could be differentially recruited in different, mutually exclusive, splicing situations. Such variable recruitment could influence the relative efficiencies with which competing, alternative splice events are carried out.

\section{Conclusions}

Our study revealed the so far uncharted evolutionary backgrounds of the $H$. sapiens B-specific protein MFAP1 and of the S. cerevisiae tri-snRNP protein Spp381. Prior to this work, MFAP1 was thought to exclusively exist in spliceosomes of complex, multicellular organisms. We have shown that an MFAP1 ortholog is present not only in S. cerevisiae but also in organisms that separated from the common lineage with complex, multicellular eukaryotes about 1.8 billion years ago. Spp381 was suggested to be one of only five yeast splicing factors without a human ortholog. Its evolutionary connection to MFAP1 reduces this number to four, raising the question if finally all ancient yeast splicing factors turn out to be conserved in complex, multicellular eukaryotes. As exemplified by the present study, identifying evolutionary connections between proteins may point to potential functions as well as potential interaction partners of poorly characterized proteins.

\section{Methods}

\section{Automated search for orthologs by InParanoid 8}

Ortholog searches were conducted using InParanoid 8 $[36,37]$. InParanoid 8 is based on sets of protein-coding genes of 273 species, where each gene is represented by one protein. These species include the 66 reference species that the 'Quest for Orthologs' community has agreed on using plus 207 additional species with completely sequenced genomes and cover all major branches of the eukaryotic tree of life (246 species) and a representative selection of 27 prokaryotes. The InParanoid methodology [38] uses a pairwise BLAST-based all-versus-all sequence comparison to detect orthologs. If candidate sequences are orthologs, they should score higher with each other than with any other sequence in the other organism's set of protein-coding genes. InParanoid further applies special cluster analysis rules to extract all in-paralogs and exclude all out-paralogs [38]. InParanoid uses a strict cut-off criterion of sequence coverage $\geq 50 \%$ and BLAST score $\geq 50$. The InParanoid 8 ortholog database $[36,37]$ provides a user interface to find orthologs inferred by the InParanoid algorithm.

Secondly, we performed RBH searches with different MFAP1 or Spp381 protein sequences against the same sets of protein-coding genes of the 273 species selected by InParanoid using the InParanoid web server [36]. A BLAST hit was considered an ortholog if the BLAST score was $\geq 30$ with E-value $\leq 0.01$, and if the reverse BLAST search, i.e. the BLAST hit was used as query in a BLAST search against the set of protein-coding genes of the original query's organism, resulted the initial query protein as the best hit. This search aims to identify orthologs that do not survive the strict cut-off criteria used for the InParanoid 8 database [37].

\section{Manual search for orthologs focused on the fungal kingdom}

For an MFAP1 ortholog search among the fungi, we performed individual BLAST searches with Homo sapiens MFAP1 (UniProt ID: P55081) as a query against the proteomes of 103 fungal species that represent the fungal tree of life as published by Medina et al. [42]. Seven MFAP1 orthologs identified in the Saccharomycotina subphylum, i.e. MFAP1 orthologs of Yarrowia lipolytica (UniProt ID: Q6CA21), Pichia pastoris (UniProt ID: A0A1B2J9D1), Debaryomyces hansenii (UniProt ID: Q6BII8), Candida albicans (UniProt ID: C4YG44), Kluyveromyces lactis (UniProt ID: Q6CJ60), Candida glabrata (UniProt ID: Q6FU95) and Saccharomyces cerevisiae Spp381 (UniProt ID: P38282), were then used as query sequences in further individual BLAST searches against the 25 Saccharomycotina species, including 14 Saccharomycetaceae species, that are part of the 103 fungal species. A BLAST hit was considered an ortholog of the query protein if the BLAST score (calculated with the BLOSUM45 scoring matrix) was $\geq 30$ with an Evalue $\leq 0.01$ and query coverage $\geq 20 \%$ (high confidence) or $\geq 10 \%$ (medium confidence), and if the reverse BLAST search resulted in the initial query protein as the best hit.

\section{Generation of multiple sequence alignment of MFAP1 orthologs}

Multiple sequence alignments of MFAP1 orthologs as shown in Fig. 3c and Additional File 4 were built with the MUSCLE algorithm (version 3.8.31; [53]) and displayed with Jalview (version 14; [54]).

\section{Pairwise sequence alignment}

Sequence identity and sequence similarity values were obtained from pairwise sequence alignments by the EMBOSS Needle tool [55] using a BLOSUM62 scoring matrix. 


\section{Protein sequence analyses}

The PredictProtein package [56] was used for secondary structure (REPROFSec), solvent exposure (PROFAcc) and structural disorder (Meta-Disorder) predictions.

\section{Plasmids for recombinant protein production in E. coli} Open reading frames (ORFs) encoding $h s \operatorname{Prp} 38$ or hsMFAP1 were amplified from a human cDNA library and cloned into the pETM11 vector using EMP cloning as described [57]. ORFs encoding $s c \operatorname{Prp} 38$ and $s c \mathrm{Spp} 381$ were PCR-amplified from $S$. cerevisiae genomic DNA and cloned into the pETM11 vector using EMP cloning [57]. Truncations and point mutations were introduced by inverse PCR as described [57]. The pETM11 vector guides the production of amino-terminally $\mathrm{His}_{6}$-tagged, TEV-cleavable fusion proteins.

\section{Protein production and purification}

Proteins bearing an $\mathrm{N}$-terminal, TEV-cleavable $\mathrm{His}_{6}$-tag were produced in E. coli Rosetta 2 (DE3) or E. coli BL21 (DE3) RIL cells in auto-inducing ZY medium [58] for $24 \mathrm{~h}$ at $18{ }^{\circ} \mathrm{C}$. The following steps were performed at $4{ }^{\circ} \mathrm{C}$. Cells were resuspended in solubilization buffer $(50 \mathrm{mM}$ sodium phosphate, $\mathrm{pH} 8.0,500 \mathrm{mM} \mathrm{NaCl}, 30 \mathrm{mM}$ imidazole, $5 \mathrm{mM} \beta$-mercaptoethanol) and lyzed using an EmulsiFlex-C5 cell homogenizer (Avestin). The soluble fraction was separated from the insoluble fraction by centrifugation for $30 \mathrm{~min}$ at 55,900 $\mathrm{x}$ g in an Avanti J-26 XP centrifuge (Beckman Coulter). Target proteins were captured on $\mathrm{Ni}^{2+}$-NTA resin (GE Healthcare), washed with solubilization buffer and eluted with elution buffer (250 mM imidazole, pH 8.0, $300 \mathrm{mM} \mathrm{NaCl}, 5 \mathrm{mM} \beta$ mercaptoethanol). Tags were cleaved with 1:50 TEV during overnight dialysis against $10 \mathrm{mM}$ sodium phosphate, $\mathrm{pH}$ 8.0, $300 \mathrm{mM} \mathrm{NaCl}, 30 \mathrm{mM}$ imidazole, $5 \mathrm{mM} \beta$ mercaptoethanol, and cleaved samples were again passed over $\mathrm{Ni}^{2+}$-NTA resin. The flow-through was collected, concentrated, and subjected to size exclusion chromatography (SEC) in SEC buffer (10 mM Tris- $\mathrm{HCl}, \mathrm{pH}$ 8.0, $300 \mathrm{mM} \mathrm{NaCl}, 0.1 \mathrm{mM}$ EDTA, $1 \mathrm{mM}$ DTT) using Superdex 75 and Superdex 200 columns (GE Healthcare). Peak fractions were analyzed by SDS-PAGE. Fractions containing the target protein were pooled, concentrated, and shock-frozen in liquid nitrogen.

\section{Analytical gel filtration chromatography}

Proteins $(50 \mu \mathrm{M})$, alone or with an equimolar amount of binding partner, were incubated in SEC buffer for $30 \mathrm{~min}$ at $4{ }^{\circ} \mathrm{C}$. $50 \mu \mathrm{l}$ of sample were analyzed on Superdex 75 PC 3.2/30 or Superdex 200 Increase 3.2/300 size exclusion columns (GE Healthcare) using an ÄKTAmicro system (GE Healthcare) at $4{ }^{\circ} \mathrm{C}$. The peak fractions were inspected by SDS-PAGE.

\section{Circular dichroism spectroscopy}

Proteins were dialyzed against CD buffer $(10 \mathrm{mM}$ sodium phosphate, $\mathrm{pH} 8.0,50 \mathrm{mM}$ sodium perchlorate) at $4{ }^{\circ} \mathrm{C}$ overnight, and diluted to a final concentration of $4.5 \mu \mathrm{M}$ $\left(h s \mathrm{MFAP}^{30-344}\right)$ or $5.1 \mu \mathrm{M}\left(s c \mathrm{Spp} 381^{\mathrm{FL}}\right)$. All spectra were recorded with a Jasco J-810 spectropolarimeter using quartz cuvettes with $0.2 \mathrm{~mm}$ path length. Initial CD spectra were collected at wavelengths between 190 and $240 \mathrm{~nm}$ at $4{ }^{\circ} \mathrm{C}$. $\mathrm{CD}$ melting profiles were then recorded by heating the samples to $90{ }^{\circ} \mathrm{C}$ at a rate of $2{ }^{\circ} \mathrm{C} / \mathrm{min}$ and following the $\mathrm{CD}$ signal at $222 \mathrm{~nm}$. Final $\mathrm{CD}$ spectra were measured at wavelengths between 190 and $240 \mathrm{~nm}$ at $90^{\circ} \mathrm{C}$.

\section{Yeast strains and yeast plasmids}

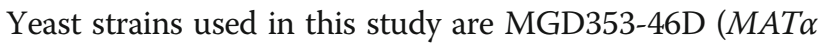
leu2-3,112 trp1-289 ura3-52 his3(-) cyh ${ }^{r}$ ) and ts192 (MATa prp38-1 leu2-3,112 trp1-289 ura3-52 his3(-) cyh'), kindly provided by Brian C. Rymond (University of Kentucky). Yeast plasmids used in this study are YEp13-2 (prp38, leu2, amp ${ }^{r}$ ), YEp13-7 (spp381, leu2, $a m p^{r}$ ), YEplac112-7A (spp381, trp1, $\left.a m p^{r}\right)$, all kindly provided by Brian C. Rymond (University of Kentucky), and YEplac112-MFAP1 (mfap1, trp1, $a m p^{r}$ ). YEplac112MFAP1 was produced by using YEplac112-7A as a template and replacing the $s c \mathrm{Spp} 381$ coding region with the coding region of $h s$ MFAP1 by EMP cloning [57].

\section{Yeast transformation}

For generation of electro-competent $S$. cerevisiae cells, a $50 \mathrm{ml}$ YPD culture was inoculated with overnight culture to an $\mathrm{OD}_{600}$ of 0.1 and grown at $30{ }^{\circ} \mathrm{C}$ and $250 \mathrm{rpm}$ to an $\mathrm{OD}_{600}$ of $1.5-10$. Cells were harvested by centrifugation for $10 \mathrm{~min}$ at $2,000 \times g$ and $4{ }^{\circ} \mathrm{C}$, resuspended in $10 \mathrm{ml} \mathrm{YPD}, 2 \mathrm{ml} 1 \mathrm{M}$ HEPES, pH 8.0, $250 \mu \mathrm{l} 1 \mathrm{M}$ DTT, and incubated for $15 \mathrm{~min}$ at $30{ }^{\circ} \mathrm{C}$ and $250 \mathrm{rpm}$. Cells were resuspended in $50 \mathrm{ml}$ of ice-cold milliQ $\mathrm{H}_{2} \mathrm{O}$ and again centrifuged for $10 \mathrm{~min}$ at $2,000 \times g, 4{ }^{\circ} \mathrm{C}$. Subsequently, cells were washed with $2 \mathrm{ml}$ ice-cold $1 \mathrm{M}$ sorbitol and centrifuged for $10 \mathrm{~min}$ at $2,000 \times g$ and $4{ }^{\circ} \mathrm{C}$. Finally, cells were resuspended in $500 \mu \mathrm{l}$ ice-cold $1 \mathrm{M}$ sorbitol, aliquoted, and directly used for transformation.

Two microgram plasmid were mixed with $50 \mu$ electrocompetent S. cerevisiae cells and incubated for $15 \mathrm{~min}$ on ice. Subsequent to the electric shock at $1,500 \mathrm{~V}, 500 \mu \mathrm{l}$ of ice-cold $1 \mathrm{M}$ sorbitol were added and cells were incubated for $2 \mathrm{~h}$ at $30{ }^{\circ} \mathrm{C}$ and $250 \mathrm{rpm}$. For selection of plasmidcontaining cells, the cell suspension was plated on minimal medium agar plates lacking leucine (in case of YEplac13 plasmids) or tryptophan (in case of YEplac112 plasmids).

\section{Yeast growth assay}

Yeast strains were grown overnight in liquid minimal medium (6.8 g/l yeast nitrogen base without amino acids, $20 \mathrm{~g} / \mathrm{l}$ glucose, $40.0 \mathrm{mg} / \mathrm{l}$ adenine, $19.2 \mathrm{mg} / \mathrm{l}$ 
uracil, $19.2 \mathrm{mg} / \mathrm{l} \mathrm{L}$-arginine, $96.0 \mathrm{mg} / \mathrm{l} \mathrm{L}$-aspartic acid, $96.0 \mathrm{mg} / \mathrm{l}$ L-glutamic acid, $19.2 \mathrm{mg} / \mathrm{l}$ L-histidine, $28.8 \mathrm{mg} / \mathrm{l} \mathrm{L}$-lysine, $19.2 \mathrm{mg} / \mathrm{l} \mathrm{L}$-methionine, $48.0 \mathrm{mg} / \mathrm{l} \mathrm{L}-$ phenylalanine, $360.0 \mathrm{mg} / \mathrm{l} \mathrm{L}$-serine, $192.0 \mathrm{mg} / \mathrm{l} \mathrm{L}$-threonine, $14.4 \mathrm{mg} / \mathrm{l}$ L-tyrosine, $144.0 \mathrm{mg} / \mathrm{l} \mathrm{L}$-valine, and for YEplac112 plasmid-containing strains $57.6 \mathrm{mg} / \mathrm{l} \mathrm{L}$-leucine, for YEp13 plasmid-containing strains $38.4 \mathrm{mg} / \mathrm{l} \mathrm{L-trypto-}$ phan, and for strains without plasmid $57.6 \mathrm{mg} / \mathrm{l} \mathrm{L}$-leucine and $38.4 \mathrm{mg} / \mathrm{l} \mathrm{L-tryptophan)} \mathrm{at} 30^{\circ} \mathrm{C}$ and $250 \mathrm{rpm}$. Subsequently, cultures were diluted to an $\mathrm{OD}_{600}$ of 2.0, 0.2, 0.02 , and 0.002. $5 \mu \mathrm{l}$ of each dilution were spotted on YPD-agar plates and plates were incubated at 23 or $37^{\circ} \mathrm{C}$ for 3 days.

\section{Additional files}

Additional file 1: Detailed results of MFAP1 and Spp381 ortholog searches with InParanoid 8. The protein sequence of Homo sapiens MFAP1 (UniProt ID: P55081) (hs), the putative Wickerhamomyces ciferrii MFAP1 ortholog (UniProt ID: KOKNQ2) (wc), or Saccharomyces cerevisiae Spp381 (UniProt ID: P38282) (sc) were used to search the InParanoid 8 [37] ortholog database and used as templates in BLAST searches against the 273 species (246 eukaryotes plus 27 prokaryotes) covered by the InParanoid 8 program. Orthologs found in the InParanoid 8 database and identified in BLAST searches against the 273 InParanoid species are marked by a black box; orthologs either found in the InParanoid 8 database or identified by the InParanoid BLAST search are marked with a grey box; species with no identified ortholog are not marked. Species names are colored according to the taxonomic group they belong to. The phylogenetic tree on the right is based on pairwise species distances derived from shared ortholog content as reported by InParanoid 8 [37]. See Additional file 2 for UniProt IDs of identified orthologs. (TIF $695 \mathrm{~kb}$ )

Additional file 2: Excel table of the results of MFAP1 and Spp381 ortholog searches with InParanoid 8. UniProt IDs of orthologs identified by BLAST searches against the 273 species covered by the InParanoid 8 program [37] and by searching the InParanoid 8 ortholog database [37] that are indicated in Fig. 1. Orthologs identified by both methods are highlighted in green; orthologs identified by one method alone are highlighted in yellow; red indicates that no ortholog was identified. (XLSX $45 \mathrm{~kb}$ )

Additional file 3: Excel table of the results of MFAP1 ortholog searches focused on the fungal kingdom. Data generated by BLAST searches against 103 fungi species (Fig. 2). UniProt IDs of potential orthologs are highlighted in green if the BLAST score (calculated with the BLOSUM45 scoring matrix) was $\geq 30$ with an E-value $\leq 0.01$ and query coverage was $\geq 20 \%$, or highlighted in orange if the BLAST score (BLOSUM 45 matrix) was $\geq 30$ with an $E$-value $\leq 0.01$ and query coverage $\geq 10 \%$. Score - BLAST scores, calculated with the BLOSUM45 scoring matrix; Coverage percentage of query sequence covered by alignment to the database sequence; E-value - Expect-value: number of hits expected to be seen by chance when searching a database of particular size; Identity - percentage of identical residues of query sequence and BLAST hit within the covered region. (XLSX $118 \mathrm{~kb})$

Additional file 4: Multiple sequence alignment of yeast MFAP1 orthologs. Multiple sequence alignment of 20 MFAP1 orthologs identified in the analyzed Saccharomycotina species. The alignment was built with the MUSCLE algorithm (version 3.8.31; [53]) and displayed with Jalview (version 14; [54]). In general, residue color intensity indicates level of sequence identity at that specific position; coloring starts at a sequence identity of 30\%. Blue - conserved hydrophobic residues; red conserved positively charged residues; purple - conserved negatively charged residues; green - conserved polar residues; cyan - conserved tyrosines or histidines; brown - conserved glycines; yellow - conserved prolines. (TIF $2772 \mathrm{~kb}$ )
Additional file 5: Excel table of the results of pairwise sequence identity/similarity analyses. The table presents sequence identity and sequence similarity values for pairs of selected MFAP1 orthologs identified in this study. The sequence identity and similarity values were obtained by the EMBOSS Needle tool [55] using a BLOSUM62 scoring matrix. (XLSX $43 \mathrm{~kb})$

\section{Abbreviations}

ca: Candida albicans; CD: Circular dichroism; ce: Caenorhabditis elegans; ct: Chaetomium thermophilum; dh: Debaryomyces hansenii; hs: Homo sapiens; kl: Kluyveromyces lactis; MFAP1: Microfibrillar-associated protein 1; pp: Pichia pastoris; Prp38: pre-mRNA processing factor 38 domain containing protein; RBH: Reciprocal best BLAST hit; RS domain: Arginine-serine-rich domain; sc. Saccharomyces cerevisiae; SDS-PAGE: Sodium dodecyl sulfate polyacrylamide gel electrophoresis; SEC: Size exclusion chromatography; snRNP: small nuclear ribonucleoprotein; Snu23: 23 kDa small nuclear ribonucleoprotein component; Spp381: pre-mRNA-splicing factor suppressor of prp38-1; SR protein: Serinearginine-rich protein; wc: Wickerhamomyces ciferrii; Y2H: Yeast two-hybrid; yl: Yarrowia lipolytica

\section{Acknowledgements}

We thank Thomas Stellwag (Freie Universität Berlin) and Patrick Knox (Beuth Hochschule für Technik) for help with cloning and purification of truncated scSpp381 and scSnu23 variants, Junqiao Jia and Ronja Janke (both Freie Universität Berlin) for advice with the yeast growth assay and Brian C. Rymond (University of Kentucky) for kindly providing yeast strains and yeast plasmids.

\section{Funding}

This work was supported by the Deutsche Forschungsgemeinschaft (grant WA $1126 / 7-1$ to MCW).

\section{Availability of data and materials}

All data generated or analyzed during this study are included in this published article and its supplementary information file.

\section{Authors' contributions}

Conceived and designed the experiments: AKCU, MCW. Performed the experiments: AKCU. Analyzed and interpreted the data: AKCU, MCW. Wrote the manuscript: AKCU, MCW. Approved the manuscript: AKCU, MCW.

\section{Competing interests}

The authors declare that they have no competing interests.

\section{Consent for publication}

Not applicable.

\section{Ethics approval and consent to participate}

Not applicable.

\section{Author details}

1'Laboratory of Structural Biochemistry, Freie Universität Berlin, Takustr. 6, D-14195 Berlin, Germany. ${ }^{2}$ Helmholtz-Zentrum Berlin für Materialien und Energie, Macromolecular Crystallography, Albert-Einstein-Straße 15, D-12489 Berlin, Germany.

Received: 11 September 2016 Accepted: 23 February 2017 Published online: 24 March 2017

\section{References}

1. Moore MJ, Sharp PA. Evidence for two active sites in the spliceosome provided by stereochemistry of pre-mRNA splicing. Nature. 1993;365(6444): 364-8.

2. Padgett RA, Grabowski PJ, Konarska MM, Seiler S, Sharp PA. Splicing of messenger RNA precursors. Annu Rev Biochem. 1986;55:1119-50.

3. Wahl MC, Will CL, Luhrmann R. The spliceosome: design principles of a dynamic RNP machine. Cell. 2009;136(4):701-18.

4. Will CL, Luhrmann R. Spliceosome structure and function. Cold Spring Harb Perspect Biol. 2011;3(7).

5. Graveley BR. Alternative splicing: increasing diversity in the proteomic world. Trends Genet. 2001;17(2):100-7. 
6. Deutsch $M$, Long M. Intron-exon structures of eukaryotic model organisms Nucleic Acids Res. 1999;27(15):3219-28.

7. Ast G. How did alternative splicing evolve? Nat Rev Genet. 2004;5(10):773-82.

8. Hawkins JD. A survey on intron and exon lengths. Nucleic Acids Res. 1988; 16(21):9893-908.

9. Berget SM. Exon recognition in vertebrate splicing. J Biol Chem. 1995;270(6): 2411-4.

10. Lim SR, Hertel KJ. Commitment to splice site pairing coincides with a complex formation. Mol Cell. 2004;15(3):477-83.

11. Michaud S, Reed R. An ATP-independent complex commits pre-mRNA to the mammalian spliceosome assembly pathway. Genes Dev. 1991;5(12B):2534-46.

12. Reed R. Mechanisms of fidelity in pre-mRNA splicing. Curr Opin Cell Biol. 2000;12(3):340-5.

13. Schneider M, Will CL, Anokhina M, Tazi J, Urlaub H, Luhrmann R. Exon definition complexes contain the tri-snRNP and can be directly converted into B-like precatalytic splicing complexes. Mol Cell. 2010;38(2):223-35

14. Bonnal S, Martinez C, Forch P, Bachi A, Wilm M, Valcarcel J. RBM5/Luca-15/ $\mathrm{H} 37$ regulates Fas alternative splice site pairing after exon definition. Mol Cell. 2008;32(1):81-95.

15. House AE, Lynch KW. An exonic splicing silencer represses spliceosome assembly after ATP-dependent exon recognition. Nat Struct Mol Biol. 2006; 13(10):937-44

16. Sharma S, Kohlstaedt LA, Damianov A, Rio DC, Black DL. Polypyrimidine tract binding protein controls the transition from exon definition to an intron defined spliceosome. Nat Struct Mol Biol. 2008;15(2):183-91.

17. Gottschalk A, Neubauer G, Banroques J, Mann M, Luhrmann R, Fabrizio P. Identification by mass spectrometry and functional analysis of novel proteins of the yeast [U4/U6.U5] tri-snRNP. EMBO J. 1999;18(16):4535-48.

18. Stevens SW, Abelson J. Purification of the yeast U4/U6.U5 small nuclear ribonucleoprotein particle and identification of its proteins. Proc Natl Acad Sci U S A. 1999;96(13):7226-31.

19. Fabrizio P, Dannenberg J, Dube $P$, Kastner B, Stark H, Urlaub H, Luhrmann R. The evolutionarily conserved core design of the catalytic activation step of the yeast spliceosome. Mol Cell. 2009;36(4):593-608.

20. Agafonov DE, Deckert J, Wolf E, Odenwalder P, Bessonov S, Will CL, Urlaub $H$, Luhrmann R. Semiquantitative proteomic analysis of the human spliceosome via a novel two-dimensional gel electrophoresis method. Mo Cell Biol. 2011;31(13):2667-82.

21. Schutze T, Ulrich AK, Apelt L, Will CL, Bartlick N, Seeger M, Weber G, Luhrmann R, Stelzl U, Wahl MC. Multiple protein-protein interactions converging on the Prp38 protein during activation of the human spliceosome. RNA. 2016;22(2):265-77.

22. Ammon T, Mishra SK, Kowalska K, Popowicz GM, Holak TA, Jentsch S. The conserved ubiquitin-like protein Hub1 plays a critical role in splicing in human cells. J Mol Cell Biol. 2014;6(4):312-23.

23. Mishra SK, Ammon T, Popowicz GM, Krajewski M, Nagel RJ, Ares Jr M, Holak TA, Jentsch S. Role of the ubiquitin-like protein Hub1 in splice-site usage and alternative splicing. Nature. 2011;474(7350):173-8.

24. Chung T, Wang D, Kim CS, Yadegari R, Larkins BA. Plant SMU-1 and SMU-2 homologues regulate pre-mRNA splicing and multiple aspects of development. Plant Physiol. 2009;151(3):1498-512.

25. Ma L, Gao X, Luo J, Huang L, Teng Y, Horvitz HR. The Caenorhabditis elegans gene mfap-1 encodes a nuclear protein that affects alternative splicing. PLoS Genet. 2012;8(7):e1002827.

26. Spartz AK, Herman RK, Shaw JE. SMU-2 and SMU-1, Caenorhabditis elegans homologs of mammalian spliceosome-associated proteins RED and fSAP57, work together to affect splice site choice. Mol Cell Biol. 2004;24(15):6811-23.

27. Spike CA, Shaw JE, Herman RK. Analysis of smu-1, a gene that regulates the alternative splicing of unc-52 pre-mRNA in Caenorhabditis elegans. Mol Cell Biol. 2001;21(15):4985-95.

28. Sugaya $K$, Hongo E, Ishihara $Y$, Tsuji $H$. The conserved role of Smu1 in splicing is characterized in its mammalian temperature-sensitive mutant. J Cell Sci. 2006;119(Pt 23):4944-51.

29. Sugaya K, Ishihara $Y$, Sugaya K. Enlargement of speckles of SF2/ASF due to loss of function of Smu1 is characterized in the mammalian temperaturesensitive mutant. RNA Biol. 2011:8(3):488-95.

30. Horrigan SK, Rich CB, Streeten BW, Li ZY, Foster JA. Characterization of an associated microfibril protein through recombinant DNA techniques. J Bio Chem. 1992;267(14):10087-95.

31. Neubauer G, King A, Rappsilber J, Calvio C, Watson M, Ajuh P, Sleeman J, Lamond A, Mann M. Mass spectrometry and EST-database searching allows characterization of the multi-protein spliceosome complex. Nat Genet. 1998 20(1):46-50.

32. Andersen DS, Tapon N. Drosophila MFAP1 is required for pre-mRNA processing and G2/M progression. J Biol Chem. 2008:283(45):31256-67.

33. Hegele A, Kamburov A, Grossmann A, Sourlis C, Wowro S, Weimann M, Will $\mathrm{CL}$, Pena $\mathrm{V}$, Luhrmann $\mathrm{R}$, Stelzl U. Dynamic protein-protein interaction wiring of the human spliceosome. Mol Cell. 2012;45(4):567-80.

34. Ulrich AK, Seeger M, Schutze T, Bartlick N, Wahl MC. Scaffolding in the spliceosome via single alpha helices. Structure. 2016;24(11):1972-83.

35. Ulrich AK, Schulz JF, Kamprad A, Schutze T, Wahl MC. Structural basis for the functional coupling of the alternative splicing factors Smu1 and RED. Structure. 2016;24(5):762-73.

36. InParanoid8. http://InParanoid.sbc.su.se. Accessed 01 Nov 2016

37. Sonnhammer EL, Ostlund G. InParanoid 8: orthology analysis between 273 proteomes, mostly eukaryotic. Nucleic Acids Res. 2015:43(Database issue): D234-9.

38. Remm M, Storm CE, Sonnhammer EL. Automatic clustering of orthologs and in-paralogs from pairwise species comparisons. J Mol Biol. 2001;314(5):1041-52.

39. Chen F, Mackey AJ, Vermunt JK, Roos DS. Assessing performance of orthology detection strategies applied to eukaryotic genomes. Plos One. 2007;2(4):e383.

40. TimeTree.org. http://www.timetree.org. Accessed 25 Jan 2017.

41. Hedges SB, Dudley J, Kumar S. TimeTree: a public knowledge-base of divergence times among organisms. Bioinformatics. 2006;22(23):2971-2.

42. Medina EM, Jones GW, Fitzpatrick DA. Reconstructing the fungal tree of life using phylogenomics and a preliminary investigation of the distribution of yeast prion-like proteins in the fungal kingdom. J Mol Evol. 2011;73(3-4): 116-33.

43. Lybarger S, Beickman K, Brown V, Dembla-Rajpal N, Morey K, Seipelt R, Rymond BC. Elevated levels of a U4/U6.U5 snRNP-associated protein, Spp381p, rescue a mutant defective in spliceosome maturation. Mol Cell Biol. 1999;19(1):577-84

44. Blanton S, Srinivasan A, Rymond BC. PRP38 encodes a yeast protein required for pre-mRNA splicing and maintenance of stable U6 small nuclear RNA levels. Mol Cell Biol. 1992;12(9):3939-47.

45. Xie J, Beickman K, Otte E, Rymond BC. Progression through the spliceosome cycle requires Prp38p function for U4/U6 snRNA dissociation. EMBO J. 1998; 17(10):2938-46.

46. Rechsteiner M, Rogers SW. PEST sequences and regulation by proteolysis. Trends Biochem Sci. 1996:21(7):267-71.

47. epestfind. http://emboss.bioinformatics.nl/cgi-bin/emboss/epestfind. Accessed 01 Nov 2016

48. Ward JJ, Sodhi JS, McGuffin LJ, Buxton BF, Jones DT. Prediction and functional analysis of native disorder in proteins from the three kingdoms of life. J Mol Biol. 2004;337(3):635-45.

49. Pace CN, Scholtz JM. A helix propensity scale based on experimental studies of peptides and proteins. Biophys J. 1998;75(1):422-7.

50. Coelho Ribeiro Mde L, Espinosa J, Islam S, Martinez O, Thanki لر Jazariegos S, Nguyen T, Larina M, Xue B, Uversky VN. Malleable ribonucleoprotein machine: protein intrinsic disorder in the Saccharomyces cerevisiae spliceosome. Peer J. 2013;1:e2.

51. Korneta I, Bujnicki JM. Intrinsic disorder in the human spliceosomal proteome. PLoS Comput Biol. 2012;8(8), e1002641.

52. Deckert J, Hartmuth K, Boehringer D, Behzadnia N, Will CL, Kastner B, Stark $\mathrm{H}$, Urlaub $\mathrm{H}$, Luhrmann $\mathrm{R}$. Protein composition and electron microscopy structure of affinity-purified human spliceosomal B complexes isolated under physiological conditions. Mol Cell Biol. 2006;26(14):5528-43.

53. Edgar RC. MUSCLE: multiple sequence alignment with high accuracy and high throughput. Nucleic Acids Res. 2004;32(5):1792-7.

54. Waterhouse AM, Procter JB, Martin DM, Clamp M, Barton GJ. Jalview Version 2-a multiple sequence alignment editor and analysis workbench. Bioinformatics. 2009;25(9):1189-91.

55. Rice P, Longden I, Bleasby A. EMBOSS: the European Molecular Biology Open Software Suite. Trends Genet. 2000:16(6):276-7.

56. Rost B, Yachdav G, Liu J. The predict protein server. Nucleic Acids Res. 2004; 32(Web Server issue):W321-6.

57. Ulrich A, Andersen KR, Schwartz TU. Exponential megapriming PCR (EMP) cloning-seamless DNA insertion into any target plasmid without sequence constraints. PLoS One. 2012;7(12):e53360.

58. Studier FW. Protein production by auto-induction in high density shaking cultures. Protein Expr Purif. 2005;41(1):207-34. 